\title{
Dynamics of a methanol-fed marine denitrifying biofilm: 2- Impact of environmental changes on the microbial community
}

\author{
Richard Villemur ${ }^{\text {Corresp., }}{ }^{,}$, Geneviève Payette ${ }^{1}$, Valérie Geoffroy ${ }^{2}$, Florian Mauffrey $^{3}$, Christine Martineau $^{4}$ \\ 1 INRS-Centre Armand-Frappier Santé et Biotechnologie, Laval, Québec, Canada \\ 2 Lallemand, Montréal, Québec, Canada \\ 3 Université de Genève, Geneva, Switzerland \\ 4 Laurentian Forestry Centre, Québec, Québec, Canada \\ Corresponding Author: Richard Villemur \\ Email address: richard.villemur@iaf.inrs.ca
}

Background. The biofilm of a methanol-fed, marine denitrification system is composed of a multi-species microbial community, among which Hyphomicrobium nitrativorans and Methylophaga

nitratireducenticrescens are the principal bacteria involved in the denitrifying activities. To assess its resilience to environmental changes, the biofilm was cultivated in artificial seawater (ASW) under anoxic conditions and exposed to a range of specific environmental conditions. We previously reported the impact of these changes on the denitrifying activities and the co-occurrence of $H$. nitrativorans strain NL23 and M. nitratireducenticrescens in the biofilm cultures. Here, we report the impact of these changes on the dynamics of the overall microbial community of the denitrifying biofilm.

Methods. The original biofilm (OB) taken from the denitrification system was cultivated in ASW under anoxic conditions with a range of $\mathrm{NaCl}$ concentrations, and with four combinations of nitrate/methanol concentrations and temperatures. The OB was also cultivated in the commercial Instant Ocean seawater (IO). The bacterial diversity of the biofilm cultures and the OB was determined by $16 \mathrm{~S}$ ribosomal RNA gene sequences. Culture approach was used to isolate other denitrifying bacteria from the biofilm cultures. The metatranscriptomes of selected biofilm cultures were derived, along with the transcriptomes of planktonic pure cultures of $H$. nitrativorans strain NL23 and $M$. nitratireducenticrescens strain GP59.

Results. High proportions of $M$. nitratireducenticrescens occurred in the biofilm cultures. $H$. nitrativorans strain NL23 was found in high proportion in the OB, but was absent in the biofilm cultures cultivated in the ASW medium at $2.75 \% \mathrm{NaCl}$. It was found however in low proportions in the biofilm cultures cultivated in the ASW medium at 0 to $1 \% \mathrm{NaCl}$ and in the 10 biofilm cultures. Denitrifying bacterial isolates affiliated to Marinobacter spp. and Paracoccus spp. were isolated. Up regulation of the denitrification genes of strains GP59 and NL23 occurred in the biofilm cultures compared to the planktonic pure cultures. Denitrifying bacteria affiliated to the Stappia spp. were metabolically active in the biofilm cultures.

Conclusions. These results illustrate the dynamics of the microbial community in the denitrifying biofilm cultures in adapting to different environmental conditions. The $\mathrm{NaCl}$ concentration is an important factor affecting the microbial community in the biofilm cultures. Up regulation of the denitrification genes of $M$. nitratireducenticrescens strain GP59 and $H$. nitrativorans strain NL23 in the biofilm cultures suggests different mechanisms of regulation of the denitrification pathway in the biofilm. Other denitrifying heterotrophic bacteria are present in low proportions, suggesting that the biofilm has the potential to

PeerJ reviewing PDF | (2019:04:36595:2:0:ACCEPTED 10 Jul 2019) 
adapt to heterotrophic, non-methylotrophic environments. 
1 Dynamics of a methanol-fed marine denitrifying biofilm: 2- Impact of

2 environmental changes on the microbial community

3

4 Authors

5 Richard Villemur ${ }^{1}$, Geneviève Payette ${ }^{1}$, Valérie Geoffroy ${ }^{1,2}$, Florian Mauffrey ${ }^{1,3}$, Christine

6 Martineau $^{1,4}$

7

8

9 Addresses

$10{ }^{1}$ INRS-Centre Armand-Frappier Santé et Biotechnologie, Laval, QC, Canada

$11 \quad{ }^{2}$ Present address: Lallemand, Montreal, QC, Canada

$12{ }^{3}$ Present address: Université de Genève, Geneva, Switzerland

$13 \quad{ }^{4}$ Present address: Laurentian Forestry Centre, Québec, QC, Canada

14

15 Corresponding author

16 Richard Villemur, richard.villemur@iaf.inrs.ca 


\section{Abstract}

Background. The biofilm of a methanol-fed, marine denitrification system is composed of a multi-species microbial community, among which Hyphomicrobium nitrativorans and Methylophaga nitratireducenticrescens are the principal bacteria involved in the denitrifying activities. To assess its resilience to environmental changes, the biofilm was cultivated in artificial seawater (ASW) under anoxic conditions and exposed to a range of specific environmental conditions. We previously reported the impact of these changes on the denitrifying activities and the co-occurrence of $H$. nitrativorans strain NL23 and $M$. nitratireducenticrescens in the biofilm cultures. Here, we report the impact of these changes on the dynamics of the overall microbial community of the denitrifying biofilm.

Methods. The original biofilm (OB) taken from the denitrification system was cultivated in ASW under anoxic conditions with a range of $\mathrm{NaCl}$ concentrations, and with four combinations of nitrate/methanol concentrations and temperatures. The OB was also cultivated in the commercial Instant Ocean seawater (IO). The bacterial diversity of the biofilm cultures and the OB was determined by $16 \mathrm{~S}$ ribosomal RNA gene sequences. Culture approach was used to isolate other denitrifying bacteria from the biofilm cultures. The metatranscriptomes of selected biofilm cultures were derived, along with the transcriptomes of planktonic pure cultures of $H$. nitrativorans strain NL23 and M. nitratireducenticrescens strain GP59.

Results. High proportions of $M$. nitratireducenticrescens occurred in the biofilm cultures. $H$. nitrativorans strain NL23 was found in high proportion in the $\mathrm{OB}$, but was absent in the biofilm cultures cultivated in the $\mathrm{ASW}$ medium at $2.75 \% \mathrm{NaCl}$. It was found however in low proportions in the biofilm cultures cultivated in the $\mathrm{ASW}$ medium at 0 to $1 \% \mathrm{NaCl}$ and in the $\mathrm{IO}$ biofilm cultures. Denitrifying bacterial isolates affiliated to Marinobacter spp. and Paracoccus spp. were isolated. Up regulation of the denitrification genes of strains GP59 and NL23 occurred in the biofilm cultures compared to the planktonic pure cultures. Denitrifying bacteria affiliated to the Stappia spp. were metabolically active in the biofilm cultures.

Conclusions. These results illustrate the dynamics of the microbial community in the 
48 denitrifying biofilm cultures in adapting to different environmental conditions. The $\mathrm{NaCl}$

49 concentration is an important factor affecting the microbial community in the biofilm cultures.

50 Up regulation of the denitrification genes of $M$. nitratireducenticrescens strain GP59 and $H$.

51 nitrativorans strain NL23 in the biofilm cultures suggests different mechanisms of regulation of

52 the denitrification pathway in the biofilm. Other denitrifying heterotrophic bacteria are present in

53 low proportions, suggesting that the biofilm has the potential to adapt to heterotrophic, non-

54 methylotrophic environments. 


\section{Introduction}

Most naturally-occurring microbial biofilms, such as those encountered in bioremediation processes, are composed of multiple microbial species. Studying such complex biofilms is a challenge, as each species can influence the biofilm development. The biofilm microbial community inside a bioremediation process adapts to the prescribed operating conditions and shapes the efficiency of the bioprocess to degrade the pollutant(s). Usually, the microbial community in such bioprocesses is complex and composed of main degraders but also of secondary microorganisms that could provide benefits to the degraders or could simply contribute to the degradation intermediates or waste. It is recognized that a complex microbial community is more resilient to "unexpected" changes in the operation of the bioprocesses than a single species biofilm, as some of the minor degraders take over the main degraders affected by the changes (Cabrol \& Malhautier 2011; Roder et al. 2016; Salta et al. 2013; Tan et al. 2017). The mechanisms of how a microbial population in a biofilm adapts to changes however are poorly understood.

The Montreal Biodome, a natural science museum, operated a continuous fluidized-bed methanol-fed denitrification reactor to remove nitrate $\left(\mathrm{NO}_{3}^{-}\right)$that accumulated in the 3 million- $\mathrm{L}$ seawater aquarium. The fluidized carriers in the denitrification reactor were colonized by naturally-occurring multispecies microorganisms to generate a marine methylotrophic denitrifying biofilm estimated to be composed of 15-20 bacterial species (Labbé et al. 2003). The main bacteria responsible of the denitrifying activities belong to the alphaproteobacteria Hyphomicrobium nitrativorans (strain representative NL23) and to the gammaproteobacteria Methylophaga nitratireducenticrescens (strain representative JAM1), both methylotrophs, that accounted for 60-80\% of the biofilm (Labbé et al. 2003; Labbé et al. 2007; Martineau et al. 2013b; Villeneuve et al. 2013).

Denitrification takes place in bacterial cells where $\mathrm{N}$ oxides serve as terminal electron acceptor instead of oxygen $\left(\mathrm{O}_{2}\right)$ for energy production when oxygen depletion occurs, leading to the production of gaseous nitrogen $\left(\mathrm{N}_{2}\right)$. Four sequential reactions are strictly required for the reduction of $\mathrm{NO}_{3}{ }^{-}$to gaseous nitrogen, via nitrite $\left(\mathrm{NO}_{2}^{-}\right)$, nitric oxide $(\mathrm{NO})$ and nitrous oxide $\left(\mathrm{N}_{2} \mathrm{O}\right)$, and each of these reactions is catalyzed by different enzymes, namely $\mathrm{NO}_{3}{ }^{-}$reductases (Nar and Nap), $\mathrm{NO}_{2}^{-}$reductases (NirS and NirK), $\mathrm{NO}$ reductases (Nor) and $\mathrm{N}_{2} \mathrm{O}$ reductases (Nos) (Kraft et al. 2011; Philippot \& Hojberg 1999; Richardson et al. 2001). Whereas H. nitrativorans 
86 strain NL23 possesses the four reductases for the complete denitrification pathway, $M$.

87 nitratireducenticrescens strain JAM1 performs incomplete denitrifying activities, as it lacks a

88 dissimilatory NO-forming nitrite reductase (Auclair et al. 2010; Martineau et al. 2013a; Mauffrey

89 et al. 2017; Mauffrey et al. 2015; Villeneuve et al. 2012). Using degenerated PCR primers for the

90 detection of denitrification genes, we showed that there are probably other denitrifying bacteria

91 in the biofilm, one to four orders of magnitude lower in proportions than $M$.

92 nitratireducenticrescens strain JAM1 and H. nitrativorans strain NL23 (Auclair et al. 2012).

93 These other bacteria may play a role if the bioprocess undergoes stress conditions or changes in 94 the operation mode.

95 We have initiated a study with the aim of assessing the performance of the Biodome 96 denitrifying biofilm subjected to environmental changes. The original biofilm (OB) taken from

97 the Biodome denitrification system was cultivated in an artificial seawater (ASW) under batch 98 mode and anoxic conditions at laboratory scale and exposed to a range of specific physico99 chemical parameters. Such parameters included a range of $\mathrm{NaCl}, \mathrm{NO}_{3}{ }^{-}, \mathrm{NO}_{2}{ }^{-}$and methanol 100 concentrations, and varying $\mathrm{pH}$ and temperatures. These parameters were chosen as possible 101 factors that could affect a denitrification reactor. Thus, the objectives of this study were to 102 determine the impact of these changes: 1) on the denitrification performance of the biofilm;2) on 103 the dynamics of the co-occurrence of $H$. nitrativorans and $M$. nitratireducenticrescens in the 104 biofilm; and 3) on the overall microbial community. The fourth objective of the study was to 105 determine whether denitrifying bacteria other than $H$. nitrativorans strain NL23 and $M$.

106 nitratireducenticrescens strain JAM1 are present in the biofilm.

107 Results for the first two objectives and partially the fourth objectives were reported by 108 Geoffroy et al. (2018) and Payette et al. (2019). We showed that the denitrifying biofilm can 109 sustain denitrifying activities in most of the tested conditions. Inhibition occurred when these 110 biofilm cultures were exposed to high $\mathrm{pH}(10)$ or to high methanol concentration (1.5\%). The

111 highest specific denitrification rates occurred when the biofilm cultures were cultivated at 64.3 $112 \mathrm{mM} \mathrm{NO}_{3}{ }^{-}$and $0.45 \%$ methanol $(\mathrm{C} / \mathrm{N}=1.5)$, and at $30^{\circ} \mathrm{C}$. Poor biofilm development occurred in

113 biofilm cultures cultivated at $5 \%$ and $8 \% \mathrm{NaCl}$. We also showed that the $\mathrm{NaCl}$ concentrations in 114 the ASW medium have significant impacts on the population of $H$. nitrativorans strain NL23, 115 with its displacement by a subpopulation of the species M. nitratireducenticrescens (strain GP59 116 as representative), which can perform the complete denitrification pathway. 
117 Results for the third and fourth objectives are presented here. The composition of the

118 bacterial community of the different biofilm cultures was determined by sequencing the $16 \mathrm{~S}$

119 ribosomal RNA (rRNA) genes. A culture dependent approach was used to recover new

120 denitrifying bacterial isolates from the biofilm cultures. To complement these two objectives, we

121 derived the metatranscriptome from selected biofilm cultures. These metatranscriptomes were

122 analyzed to determine the composition of the active microbial community in the biofilm cultures

123 but also to assess their metabolic contributions, such as those involved in denitrification.

124 Furthermore, metatranscriptomic analyses provided further indications on the dynamics of $H$.

125 nitrativorans and $M$. nitratireducenticrescens in these cultures (second objective) by assessing

126 changes in metabolic pathways of $H$. nitrativorans strain NL23 and M. nitratireducenticrescens

127 strain GP59 between the planktonic pure cultures and the biofilm cultures. Our study is the first

128 that give a comprehensive picture of the microbial community of a methylotrophic denitrifying

129 biofilm and its adaptation to specific changes.

130

131 Material and Methods

132

\section{Cultivation of the original biofilm to different culture conditions}

134 The formulations of the artificial seawater (ASW) medium and the commercial Instant

135 Ocean (IO) medium (Table S1), and the different conditions of the biofilm cultures were

136 described by Payette et al. (2019). Briefly, the biomass of several carriers taken from the

137 denitrification reactor of the Montreal Biodome was scraped, dispersed, then distributed to

138 several vials containing twenty free carriers and $60 \mathrm{~mL}$ prescribed medium (Table 1; Fig. S1).

139 The vials were incubated under anoxic conditions at $23^{\circ} \mathrm{C}$ or $30^{\circ} \mathrm{C}$ (Table 1) and shaken at 100

$140 \mathrm{rpm}$ (orbital shaker). In average once a week, the twenty carriers were taken, gently washed to

141 remove the excess medium and the planktonic bacteria, then transferred into fresh anoxic

142 medium and incubated under the same conditions (Fig. S1). The Ref300N-23C biofilm cultures

143 (for $300 \mathrm{mg} \mathrm{NO}_{3}^{-}-\mathrm{N} / \mathrm{L}, 23^{\circ} \mathrm{C}$ ) were defined as the reference biofilm cultures. These cultures were

144 used by Payette et al. (2019) as a reference to compare results between the different culture

145 conditions. The protocols to measure $\mathrm{NO}_{3}{ }^{-}$and $\mathrm{NO}_{2}{ }^{-}$concentrations, and to extract DNA from

146 the biofilm cultures or the planktonic pure cultures were described in Payette et al. (2019) and

147 Geoffroy et al. (2018). 


\section{S rRNA gene analysis}

DNA extracted from triplicate biofilm cultures was pooled before sequencing. Total DNA samples from seven biofilm cultures (Table 1; Ref300N-23C, 300N-30C, 900N-23C, 900N-30C, $0 \% \mathrm{NaCl}, 0.5 \% \mathrm{NaCl}$ and $1.0 \% \mathrm{NaCl}$ ) were sent to the sequencing service at the Research and

153 Testing Laboratory (RTL, Lubbock, Texas, USA). A region of the 16S rRNA gene was PCR

154 amplified using the 28F-519R primers (5' GAGTTTGATCNTGGCTCAG 3' and 5'

155 GTNTTACNGCGGCKGCTG 3', covering the V1-V2-V3 variable regions) and subjected to pyrosequencing using a Roche 454 FLX genome sequencer system. The sequencing service

157 (RTL) performed denoising and chimera analyses (details provided in supplemental doc 1). The high-quality reads were then processed in the RDP pipeline at the Ribosomal data project (RDP) web site (Cole et al. 2014). Reads were clustered into operational taxonomic units (OTU) using a 97\% identity threshold. DNA extracted from the OB (from frozen stock) and from fresh IO biofilm cultures (Table 1) were sent to the sequencing service of Genome Quebec Innovation Center (Montreal, QC, Canada). In these cases, the 16S rRNA sequences covering the V6-V7-V8 variable regions (5' ACACTGACGACATGGTTCTACA 3' and 5'

TACGGTAGCAGAGACTTGGTCT 3') were PCR amplified and sequenced by Illumina MiSeq PE250 (250 bp paired-end sequencing reactions). The reads were processed based on Peck et al. (2016). Briefly, paired-end reads were merged with minimum and maximum overlap length between the two reads of 20 and 250 bases, respectively, with $30 \%$ mismatched bp tolerance in the overlap region. The merged reads were processed using the software UPARSE (Edgar 2013). Sequences were truncated to a uniformized length to $420 \mathrm{bp}$. Reads with a low-quality score were removed using 2.0 as the maximum expected error value. The high-quality reads were dereplicated, sorted by size and singletons were removed. The resulting reads were clustered into operational taxonomic units (OTU) with the UPARSE-OTU clustering method using a 97\%

173 identity threshold. Chimeric OTU were removed by UPARSE-REF algorithm

174 and with the software UCHIME ran against ChimeraSlayer 'gold' reference database (Edgar et 175 al. 2011). All representative sequences of the OTUs (from pyrosequencing and Illumina) were

176 checked again for chimeras with the DECIPHER v 2.0 program

177 (http://www2.decipher.codes/FindChimeras.html) (Wright et al. 2012). The affiliation of the 178 OTUs to the most probable genus was determined by the CLASSIFIER program at the RDP web 
179 site (Supplemental doc 2). 16S rRNA sequence reads were deposited in the GenBank Sequence

180 Read Archive (SRA) under the accession number PRJNA524642. Principal component analysis

181 of the proportion of reads associated to the bacterial profiles were performed at ClustVis web site

182 (https://biit.cs.ut.ee/clustvis/) (Metsalu \& Vilo 2015).

183

184 Isolation of bacterial isolates

185 Biofilm of the Ref300C-23C biofilm cultures was scraped from the carriers and dispersed 186 in saline solution $(3 \% \mathrm{NaCl}, 34.2 \mathrm{mM}$ phosphate buffer $\mathrm{pH} 7.4)$, and serial dilutions were made 187 and inoculated onto these agar plate media: (1) R2A medium (complex organic carbons; EMD

188 Chemicals Inc., Gibbstown, NJ, USA), (2) Marine Agar 2216 (marine medium with yeast extract 189 and peptone as carbon source; Becton, Dickinson and Co., Sparks, MD, USA), (3) Methylophaga 190 medium 1403 (American Type Culture Collection [ATCC], Manassas, VA, USA) and (4) the 191 ASW medium; these two latter media were supplemented with $1.5 \%$ agar and $0.3 \% \mathrm{v} / \mathrm{v}$

192 methanol. The isolation procedure, the taxonomic affiliation of the isolates and the measurement 193 of their denitrifying activities were carried out as described by Geoffroy et al. (2018). The 16S 194 rRNA gene sequences were deposited in GenBank under the accession numbers MK571459 to 195 MK571476.

\section{Transcriptomes}

Planktonic pure cultures of $M$. nitratireducenticrescens strains JAM1 and GP59 were performed in the Methylophaga 1403 medium and of $H$. nitrativorans strain NL23 in the 337a medium as described by Martineau et al. (2015) and Mauffrey et al. (2015). These cultures were carried out in triplicate with methanol $(0.3 \%)$ and $\mathrm{NO}_{3}{ }^{-}(21.4 \mathrm{mM}[300 \mathrm{mg}-\mathrm{N} / \mathrm{L}])$ under anoxic conditions at $30^{\circ} \mathrm{C}$. The biomass was collected by centrifugation when the $\mathrm{NO}_{3}{ }^{-}$reduction was near completion, and total RNA was extracted as described by Mauffrey et al. (2015). For the biofilm cultures, at the end of the the fifth transfer cultures, the biomass of each replicate was scrapped from carriers and used to extract total RNA. The RNA samples were sent to the sequencing service for RNA sequencing (RNAseq) by Illumina (Genome Quebec Innovation Center, Montreal QC, Canada). Because of limited amount of biofilm available, total RNA from the triplicate biofilm samples were pooled before sending to the sequencing service. For the planktonic pure cultures, RNAseq was performed on each replicate. The Ribo-Zero ${ }^{\mathrm{TM}}$ rRNA 
210 Removal Kit (Meta-Bacteria; Epicentre, Madison, WI, USA) was used to deplete total RNA of

211 the ribosomal RNA. The RNA was then treated with the TruSeq Stranded mRNA Sample Prep

212 Kit (Illumina Inc, San Diego, CA, USA).

213 All computations were made on the supercomputer Briarée from the Université de

214 Montréal, managed by Calcul Québec and Compute Canada. Raw reads were filtered to remove

215 low quality reads using FASTX toolkit (http://hannonlab.cshl.edu/ fastx_toolkit/) by discarding

216 any reads with more than $10 \%$ nucleotides with a PHRED score $<20$. The resulting reads from

217 each sample/replicate were aligned respectively to the genome of $M$. nitratireducenticrescens

218 strain JAM1 (GenBank accession number CP003390.3), to the genome and plasmids of $M$.

219 nitratireducenticrescens strain GP59 (CP021973.1, CP021974.1, CP021975.1) and to the

220 genome of H. nitrativorans strain NL23 (CP006912.1) using Bowtie (v 2.2.3) with default

221 parameters. SAMtools (v 0.1.18) and BEDtools (v 2.20.1) were used for the generation of sam

222 and bam files, respectively. Significance for difference in the relative transcript levels of a gene

223 (defined as transcript per million: TPM) between planktonic pure cultures and biofilm cultures

224 was performed with the R Bioconductor NOIseq package v2.14.0 (NOIseqBio) (Tarazona et al.

225 2011) and run with the R software v3.2.3 (Team 2015). Because the RNAseq from the biofilm

226 samples were derived from one pooled RNA preparation, the "no replicate parameter" was set

227 ( $p n r=0.2, n s s=5$ and $v=0.02$; pseudoreplicate generated) in NOIseq as described by Tarazona et

228 al. (2011) under the NOISeq-sim section. Briefly, NOISe1-sim assumes (quoting) "that read

229 counts follow a multinomial distribution, where probabilities for each gene in the multinomial

230 distribution are the probability of a read to map to that gene". Results from this statistical

231 analysis showed that genes that had at least $>2$-fold higher transcript levels from one type of

232 cultures to the other showed significant differences. RNAseq reads from the planktonic pure

233 cultures and the biofilm cultures were deposited in the SRA under the accession number

234 PRJNA525230. Annotations were based on services provided by GenBank

235 (https://www.ncbi.nlm.nih.gov/genbank), RAST (Rapid Annotation using Subsystem

236 Technology; http://rast.nmpdr.org) and KEGG (Kyoto Encyclopedia of Genes and Genomes;

237 https://www.genome.jp/kegg) (Supplemental doc 3).

238 To derive transcript reads not associated to the $M$. nitratireducenticrescens and $H$.

239 nitrativorans, reads were aligned to a concatenated sequence consisting of the three reference

240 genomes (JAM1+GP59+NL23) and the two plasmids (from strain GP59). The reads that did not 
241 align were kept. Unaligned reads were de novo assembled at the National Center for Genome

242 Analysis web site (https://galaxy.ncgas-trinity.indiana.edu) by Trinity v. 2.4.0 (Grabherr et al.

243 2011). These transcripts were deposited in SRA under the accession number PRJNA525230.

244 Estimation of the transcript abundance of the de novo assembled sequences was performed by

245 RSEM (Li \& Dewey 2011). The resulting assembled sequences were annotated at the Joint

246 Genomic Institute (https://img.jgi.doe.gov/cgi-bin/m/main.cgi) to find open reading frames with

247 their putative function and affiliation (GOLD Analysis Project Id: Ga0307915, Ga0307877,

$248 \mathrm{Ga} 0307760)$. The annotations were then verified manually for discrepancies within the

249 assembled sequences (Supplemental doc 4, 5, 6 and 7).

250

251

\section{Results}

252

253

\section{Bacterial composition of the biofilm cultures by 16S rRNA gene sequencing}

As reported by Payette et al. (2019), the original biofilm (OB) collected from the Biodome

255

denitrification system was used as inoculum to colonize new carriers in a series of anoxic biofilm

256

cultures cultivated under different conditions (Table 1; Fig. S1). We selected eight of these

257 biofilm cultures for our present analysis for the following reasons. The Ref300N-23C biofilm cultures were used as reference for comparison analysis. The $300 \mathrm{~N}-30 \mathrm{C}, 900 \mathrm{~N}-23 \mathrm{C}$ and $900 \mathrm{~N}-$ $30 \mathrm{C}$ biofilm cultures had higher specific denitrification rates compared to the Ref300N-23C biofilm cultures. The $0 \%, 0.5 \%$ and $1.0 \% \mathrm{NaCl} \mathrm{ASW}$ biofilm cultures and the IO biofilm cultures were chosen because of the persistence of $H$. nitrativorans NL23 in these cultures as opposed to the other cultures. The composition of the bacterial community of these biofilm cultures and the $\mathrm{OB}$ was determined by sequencing the $16 \mathrm{~S}$ rRNA genes to assess the impact of these specific conditions on the bacteria community (Fig. 1A; Table 2). The bacterial profiles of the $\mathrm{OB}$ and the IO biofilm cultures were distinct to each other, and to the seven biofilm cultures cultivated with different formulations in the ASW medium (Fig. 1B). The bacterial profiles of these latter cultures were however not very different because of the high proportions of Methylophaga spp. (>85\%).

269 In the OB, high proportions of the $16 \mathrm{~S}$ rRNA gene sequences were related to Hyphomicrobium spp. (45.8\%) followed by Oceanibaculum spp. (12.3\%), Aquamicrobium spp. 
272 proportion of Methylophaga spp. was 12 times higher (42.8\%) than in the OB, whereas it was 5.5 273 times lower for Hyphomicrobium spp. (8.4\%). Higher proportions of Marinicella spp. (7.2\%)

274 and Winogradskyella spp. (4.3\%) along with a much lower proportion of Aquamicrobium spp.

275 (0.44\%) were observed in the IO biofilm cultures compared to the OB (Table 2).

276 In the biofilm cultures cultivated with the four combinations of $\mathrm{NO}_{3}^{-} /$methanol

277 concentrations and temperatures in ASW medium containing 2.75\% $\mathrm{NaCl}$ (Ref300N-23C, 300N-

278 30C, 900N-23C, 900N-30C), Methylophaga spp. accounted for $>90 \%$ of the $16 \mathrm{~S}$ rRNA gene

279 sequences followed by Marinicella spp. with proportions ranging from $1.5 \%$ to $5.0 \%$ (Fig. 1;

280 Table 2). No sequences were found affiliated to Hyphomicrobium spp. under these conditions. In

281 the biofilm cultures cultivated at low $\mathrm{NaCl}$ concentrations $(0 \% \mathrm{NaCl}, 0.5 \% \mathrm{NaCl}, 1 \% \mathrm{NaCl})$,

282 Hyphomicrobium spp. accounted for $11.8 \%, 6.8 \%$ and $0.25 \%$, respectively of the $16 \mathrm{~S}$ rRNA

283 gene sequences (Fig. 1; Table 2). Methylophaga spp. was still the dominant genus with more

284 than $85 \%$ of the $16 \mathrm{~S}$ rRNA gene sequences; $16 \mathrm{~S}$ rRNA gene sequences affiliated to Marinicella

285 spp. were also found in significant proportions (Fig. 1; Table 2). Finally, 16S rRNA gene

286 sequences affiliated to Stappia spp. were found in all biofilm cultures and in the OB.

287 The 16S rRNA gene sequences from the OB and the IO biofilm cultures that were derived

288 by Illumina sequencing generated several thousands of reads affiliated to Hyphomicrobium spp.

289 and Methylophaga spp. (Table 3). This tremendous amounts of sequences allowed assessing the

290 presence of species other than H. nitrativorans and M. nitratireducenticrescens in these two

291 biofilms. The phylogenic analyses performed on these sequences allowed regrouping the OTUs

292 in three clusters for Hyphomicrobium spp., and also three clusters for Methylophaga spp. (Fig.

293 S2A and S2B). The vast majority ( $>90 \%$ ) of the $16 \mathrm{~S}$ rRNA gene sequences associated to these

294 OTUs were affiliated to H. nitrativorans or M. nitratireducenticrescens, respectively, in the OB

295 and the IO biofilm cultures (Clusters 1, Table 3). A small proportion of the OTUs (clusters 2 and

296 3) was affiliated to other Hyphomicrobium or Methylophaga, which suggests that other members

297 of these genera were present in these biomasses in very low proportions.

298

299 Isolation of denitrifying bacterial isolates from the biofilm cultures

300 The biomass of the Ref300N-23C biofilm cultures was dispersed on different nutrient agar

301 plates to isolate denitrifying bacteria other than H. nitrativorans strain NL23 and M.

302 nitratireducenticrescens strain GP59. Isolates affiliated to the genera Marinobacter, 
303 Pseudomonas, Paracoccus, Roseovarius, Thalassobius, Winogradskyella, Aequorivita and

304 Exiguobacterium (Table 4) were recovered from the Marine medium 2216 plates, which contains

305 yeast extract and peptone (Atlas 1993). Only isolates affiliated to the genera Marinobacter and

306 Paracoccus showed consumption of $\mathrm{NO}_{3}{ }^{-}$and $\mathrm{NO}_{2}{ }^{-}$and production of gas, suggesting that they

307 possess the complete denitrification pathway. The three isolates affiliated to the Paracoccus spp.

308 have identical 16S rRNA sequences with the one of Paracoccus sp. strain NL8 that was isolated

309 from the Biodome denitrification system (Labbé et al. 2003). This result suggests that strain NL8

310 persisted in the biofilm cultures. One representative of Paracoccus isolates (GP3) could grow

311 with methanol as sole source of carbon; Marinobacter sp. GP2 could not.

312

\section{Metatranscriptomic analysis of the biofilm cultures}

314 The metatranscriptomic approach has allowed assessing the contributions of the microbial

315 community to the metabolic processes in the biofilm cultures. We have chosen to focus on three

316 biofilm cultures, which were the Ref300N-23C (the reference biofilm cultures), 900N-30C

317 (highest denitrification rates; Table 1) and $0 \% \mathrm{NaCl}$ (persistence of $H$. nitrativorans strain NL23)

318 biofilm cultures. Because the genomes of H. nitrativorans strain NL23 and M.

319 nitratireducenticrescens strain JAM1 and strain GP59 were available, we first determined

320 changes in the transcript levels of genes associated to these genomes between the biofilm

321 cultures and the planktonic pure cultures of the respective strains. To assess the contribution of

322 other microorganisms in biofilm cultures, the reads from the metatranscriptomes that did not

323 align with the three reference genomes were used to derive de novo assembled transcripts. These

324 transcripts were annotated for function and bacterial affiliation.

325

326 Gene expression profiles of $M$. nitratireducenticrescens strain GP59 in the biofilm cultures

327 Because $>80 \%$ of the genomes of strains JAM1 and GP59 are identical, high proportions

328 of reads from the biofilm metatranscriptomes can align to both genomes. Geoffroy et al. (2018)

329 showed that the gene expression profiles of the common genes between both strains in

330 planktonic pure cultures were similar. In Payette et al. (2019), the concentrations of strain GP59

331 and strain JAM1 in the biofilm cultures were determined by qPCR. In the three selected biofilm

332 cultures for the metatranscriptomic analysis, the concentrations of strain GP59 (copies of nirK by

333 ng biofilm DNA) were one to three orders of magnitude higher than those of strain JAM1 (copies 
334 of $\operatorname{tag} H$ by ng biofilm DNA). Because of these differences, it was assumed that most of the

335 transcript reads associated to M. nitratireducenticrescens in the biofilm cultures were from strain

336 GP59. Metatranscriptomic analysis in relation with strain JAM1 is described in supplemental doc

3378 and 11. The transcriptomes of strain GP59 were also derived from planktonic pure cultures

338 cultivated under anoxic conditions in the Methylophaga 1403 medium (Fig. S1). The choice of

339 this medium was because suboptimal growth occurred with strain GP59 in ASW medium. The

340 relative transcript levels of the corresponding genes in the biofilm cultures and the planktonic

341 pure cultures were compared to assess changes in the metabolisms of the strain that occurred

342 between the two environments. All quantitative changes described below of the transcript levels

343 in the biofilm cultures are expressed relative to the transcript levels in the planktonic pure

344 cultures.

345 Among all genes of strain GP59, between $11 \%$ and $21 \%$ of them had higher relative

346 transcript levels in the biofilm cultures. At the opposite, 6 to 17\% of all genes of strain GP59

347 were expressed at higher relative transcript levels in planktonic pure cultures (Fig. 2). Strain

348 GP59 contains two plasmids, and most of the genes encoded by these plasmids had much lower

349 relative transcript levels in the biofilm cultures (Fig. 2). Genes involved in the nitrogen

350 metabolism and iron transport were globally at higher relative transcript levels in the biofilm

351 cultures (Table 5; Fig. 2 and 3).

352 For the denitrification genes, narXL encoding the regulatory factors of the nar systems

353 showed no differences between the biofilm cultures and the planktonic pure cultures in the

354 relative transcript levels (Table 5). Small upregulation of the nar 2 operon with about 3 -fold

355 increases in relative transcript levels occurred in the biofilm cultures. These levels were lower in

356 the $900 \mathrm{~N}-30 \mathrm{C}$ biofilm cultures for the narl operon and were about the same levels in the two

357 other biofilm cultures and the planktonic pure cultures. The norl operon had the same relative

358 transcript levels in the $300 \mathrm{~N}-23 \mathrm{C}$ and $900 \mathrm{~N}-30 \mathrm{C}$ biofilm cultures and the planktonic pure

359 cultures, and a 3-fold decrease was noticed in these levels in the $0 \% \mathrm{NaCl}$ biofilm cultures (Table

360 5). No significant changes in the expression of the nos operon occurred between both types of

361 cultures. The relative transcript levels of nirK were 5 to 10 -times higher in the $300 \mathrm{~N}-23 \mathrm{C}$ and

$362900 \mathrm{~N}-30 \mathrm{C}$ biofilm cultures, whereas these levels were similar in the $0 \% \mathrm{NaCl}$ biofilm cultures

363 and the planktonic pure cultures (Table 5). Higher relative transcript levels of genes involved in

364 the ammonium transport and the assimilatory $\mathrm{NO}_{3}{ }^{-} / \mathrm{NO}_{2}{ }^{-}$reduction pathway were observed in the 
365 biofilm cultures (Table 5; Fig. 3). Absence of nitrogen source other than $\mathrm{NO}_{3}{ }^{-}$in the ASW 366 medium and presence of $37 \mathrm{mM} \mathrm{NH}_{4}{ }^{+}$in the medium used for the planktonic pure cultures

367 (Methylophaga 1403) could explain these differences in the assimilatory pathway.

368 Figure 3 illustrates changes in the relative expression profiles in the biofilm cultures of

369

370

371

372

373

374

375

376

377

378

379

380

381

382

383

384

385

386

387

388

389

390

391

392

393

394

395 major pathways in strain GP59. The relative transcript levels of mxaFJGI encoding the small and large subunits of the methanol dehydrogenase (MDH) and the cytochrome c-L increased by 2-6 fold in biofilm cultures. Two out of the four $m x a F$-related products $(x o x F)$ showed 2- to 9-fold decreases in their relative transcript levels in the biofilm cultures. As observed in Methylorubrum extorquens, the genome of $M$. nitratireducenticrescens strain GP59 encodes three formate dehydrogenases with the same gene arrangement (Chistoserdova et al. 2004). The $f d h C B A D$ operon that encodes the NAD-linked, Mo-formate dehydrogenases showed ca. 60-fold increases in the relative transcript levels in the biofilm cultures, whereas the two other $f d h$ operons stayed at the same levels of those of the planktonic pure cultures. Gene encoding NAD-dependent formate dehydrogenase was upregulated in biofilms formed by Desulfovibrio vulgaris compared to planktonic cultures (Clark et al. 2012). This was also the case in biofilms formed by

Staphylococcus aureus where the NAD-dependent formate dehydrogenase gene was among the highest upregulated genes (Resch et al. 2005). In both studies, this upregulation correlated with increase in formate dehydrogenase activity. Contrary to planktonic cultures, accumulation of formate could occur in cell vicinity in the biofilm that would be toxic for the cells (Resch et al. 2005). Therefore, upregulation of $f d h C B A D$ operon could be related to detoxification. The relative transcript levels of the gene encoding the cytochrome c555 were 40 to 70 times higher in the biofilm cultures. Genes encoding the other cytochromes, pseudoazurins and azurin were expressed at similar levels in both types of cultures. Genes involved in the formaldehyde metabolism to formate and $\mathrm{CO}_{2}$, glycolysis, the ribulose monophosphate pathway, the Entner Doudorof pathway, the tricarboxylic acid cycle, and the pentose pathway showed their relative transcript levels in general unchanged. Few genes in these pathways had 2-11 fold differences between the planktonic pure cultures and the biofilm cultures. The genome of strain GP59 encodes the major enzymes involved in the Calvin-Benson-Bassham cycle: the ribulosebisphosphate carboxylase (Rubisco) and the phosphoribulokinase (Prk). In the $0 \% \mathrm{NaCl}$ biofilm cultures, the relative transcript levels of the Rubisco gene operon ( $r b c S L)$ jumped by 66 times. This upregulation was less pronounced in the Ref $300 \mathrm{~N}-23 \mathrm{C}$ biofilm cultures (3-fold increase). 
396 For the prk gene, the relative transcript levels were 3 to 4 times higher in the $0 \% \mathrm{NaCl}$ biofilm

397 cultures. The nature of this upregulation is unknown. Except for the cytochrome c555, the

398 relative transcript levels of genes encoding for the oxidative phosphorylation metabolism were

399 unchanged. Several genes involved in iron transport showed higher relative transcript levels in

400 the biofilm cultures ( 2 to $>50$-fold increases). The nature of this upregulation in the biofilm

401 cultures is unknown, as the biofilm and planktonic pure cultures were cultivated with trace

402 elements containing iron (Payette et al. 2019).

403

404

Gene expression profiles of $H$. nitrativorans strain $\mathrm{NL23}$ in the $0 \% \mathrm{NaCl}$ biofilm cultures

405

As with M. nitratireducenticrescens strain GP59, the transcript levels of genes associated

406

to H. nitrativorans strain NL23 were compared between the biofilm cultures and the planktonic

407

pure cultures to assess changes in the metabolisms that occurred between the two environments.

408

The planktonic pure cultures of strain NL23 were cultivated under anoxic conditions in the 337a

409

medium (Fig. S1). The choice of this medium was because strain NL23 could not grow in ASW

410 (with $2.75 \% \mathrm{NaCl}$ ).

411 The overall analysis of the three metatranscriptomes confirmed the results obtained by

412 qPCR assays (Payette et al. 2019) and the 16S rRNA gene analysis (Table 2). High number of

413 reads $\left(40 \times 10^{6}\right)$ derived from the metatranscriptome of the $0 \% \mathrm{NaCl}$ biofilm cultures aligned

414 with the NL23 genome, but $<20000$ reads derived from the Ref300N-23C and $900 \mathrm{~N}-30 \mathrm{C}$

415 metatranscriptomes did. In the $0 \% \mathrm{NaCl}$ biofilm cultures, $<10 \%$ of all NL23 genes had a higher

416 relative transcript levels in the $0 \% \mathrm{NaCl}$ biofilm cultures, whereas this was the case for $>40 \%$

417 genes in planktonic pure cultures (Fig. 2). These results suggest important changes had occurred

418 in the regulation of gene expression between the planktonic pure cultures and the biofilm

419 cultures. Genes involved in the energy (Fig. S4) and nitrogen metabolisms (Fig. 2; Table 5) had

420 globally higher relative transcript levels in the $0 \% \mathrm{NaCl}$ biofilm cultures (Fig. 2).

$421 \quad$ Higher relative transcript levels (5 to 8-times) for the nap, nor and nos operons were

422 observed in the $0 \% \mathrm{NaCl}$ biofilm cultures (Table 5). nirK was highly upregulated in the biofilm

423 cultures with 49-fold increase in the relative transcript levels (Table 5). The napGH operon

424 however had a 9.4-fold decrease in the relative transcript levels in the $0 \% \mathrm{NaCl}$ biofilm cultures.

425 As observed with strain GP59, substantial changes in the relative transcript levels of genes

426 involved in the ammonium transport and the assimilatory $\mathrm{NO}_{3}{ }^{-}-\mathrm{NO}_{2}{ }^{-}$reductase were observed 
427 with 3- to 22-fold increases in these levels (Table 5, Fig. 4). These results correlate with the 428 absence of $\mathrm{NH}_{4}{ }^{+}$in the ASW medium, and thus $\mathrm{NO}_{3}{ }^{-}$the only source of $\mathrm{N}$, compared to the $337 \mathrm{a}$ medium used for the planktonic pure cultures, which contains $3.8 \mathrm{mM} \mathrm{NH}_{4}{ }^{+}$.

$430 \quad$ Figure 4 illustrates changes in relative expression profiles of major pathways in the $0 \%$ $431 \mathrm{NaCl}$ biofilm cultures of strain NL23. The relative transcript levels of mxaFJGI increased by 2432 fold in the biofilm cultures. The relative transcript levels of the mau operon (methylamine 433 dehydrogenase) showed a 15 -fold decrease in the biofilm cultures. The nature of such decrease is 434 unknown as strain NL23 was not fed with methylamine in any of our cultures. The three $\operatorname{xoxF}$ 435 genes did not show substantial changes in their transcript levels in both types of cultures. Genes 436 involved in the formaldehyde metabolism to formate and $\mathrm{CO}_{2}$, glycolysis, the tricarboxylic acid 437 cycle, and the pentose pathway showed their transcript levels in general unchanged between the 438 planktonic pure cultures and the biofilm cultures. Few genes in these pathways had 2-5 fold 439 differences in their relative transcript levels. The two genes encoding the key enzymes in the 440 serine pathway (alanine-glyoxylate transaminase, glycine hydroxymethyltransferase) had 3- to 7441 fold increases in their relative transcript levels in the biofilm cultures. As Methylorubrum 442 extorquens, the NL23 genome encodes the ethymalonyl-CoA pathway (Chistoserdova et al. 443 2003; Peyraud et al. 2009), which did not show changes overall in the transcript levels of the 444 corresponding genes between the two types of cultures. Contrary to M. extorquens however, a 445 gene encoding the isocitrate lyase is present in strain NL23 and showed a 25-fold upregulation in 446 the biofilm cultures. The isocitrate lyase is one of the key enzymes of the glyoxylate bypass that 447 catalyzes the transformation of isocitrate to succinate and glyoxylate. Gene encoding isocitrate 448 lyase is also present in other available Hyphomicrobium genomes. All these results suggest that 449 in the $0 \% \mathrm{NaCl}$ biofilm cultures, the carbon metabolism increased in activity and that the glycine 450 regeneration for the serine pathway by the glyoxylate was upregulated. Among genes involved in 451 the oxidative phosphorylation, the relative transcript levels were higher (2 to 13 times) in the 452 biofilm cultures with those encoding the NADH dehydrogenase, the cytochrome c reductase, 453 with one of the cytochromes $\mathrm{c}$ and the F-type ATPase. Combined with increases in the relative 454 transcript levels of the denitrification and the carbon pathways, these results suggest that 455 increases in electron donor activities correlates with the need of electron for the nitrogen 456 dissimilatory metabolism in the biofilm cultures. Strain NL23 possesses four types of 457 cytochrome oxidase (aa3, bo, bd-I and cbb3) (reduction of $\mathrm{O}_{2}$ in $\mathrm{H}_{2} \mathrm{O}$ ) that are in general 
458 expressed at the same levels in both types of cultures. Figure 4 also illustrates the dynamic 459 changes of transporters and two component systems. Several of these transporters had lower 460 relative transcript levels in the $0 \% \mathrm{NaCl}$ biofilm cultures. Contrary to strain $\mathrm{GP} 59$, genes 461 involved in iron transport were not strongly affected in their gene expression in the $0 \% \mathrm{NaCl}$ 462 biofilm cultures (Fig. 2 and 4).

463

\section{The composition of the active microbial community in the biofilm cultures}

As mentioned above, the reads from the three metatranscriptomes that did not align with the genomes of $H$. nitrativorans strain NL23 and M. nitratireducenticrescens strain GP59 and strain JAM1 were de novo assembled. These reads were subsequently aligned to the de novo assembled transcripts to derive the relative levels of these transcripts in the biofilm cultures. The de novo assembled sequences were then annotated for function and affiliation. Finally, these sequences were grouped by microbial affiliation to determine the active populations in the biofilm cultures and to assess their level of involvement in these biofilm cultures (Table 6).

It was estimated that between 5 to $10 \%$ reads of the three metatranscriptomes were derived from other microorganisms than H. nitrativorans strain NL23 and M. nitratireducenticrescens strain GP59 and strain JAM1 (Supplemental doc 4). The proportions of transcripts affiliated to Archaea and Eukarya accounted together for $<0.1 \%$ (Table 6), which suggests very low abundance of these microorganisms in the biofilm cultures. The proportions of transcripts affiliated to viruses, phages and plasmids in the de novo assembled transcripts represented between 0.6 and $21.7 \%$ (Table 6 ).

Twenty-seven bacterial taxa were selected for their overall transcript levels in at least one of the three biofilm cultures (Table 6). All the taxa detected by the 16S rRNA gene sequencing are present in this list (Table 2 and supplemental doc 2). These 27 taxa represented between 22\% and $35 \%$ of the de novo assembled transcripts. Among these taxa, genes encoding the four denitrification reductases were present in the de novo transcripts affiliated to Marinobacter spp., Stappia spp. and Pseudomonas spp. However, only de novo assembled transcripts affiliated to the Stappia spp. showed the complete set of denitrification genes in the three biofilm cultures and organized in operons (napABC, napADFE, norCBQD, nosRZDF).

Further analysis of the expression profiles of the 27 bacterial taxa was performed to assess 
489 of the biofilm cultures. The overall transcript levels of the 27 bacterial taxa (Table 6) were

490 compared between each biofilm culture by clustering analysis (Fig. 5). $\mathrm{NaCl}$ concentration was

491 the main factor of clustering as two distinct clusters were derived. The low salt cluster consisting

492 of nine bacterial taxa showed higher relative transcript levels in the $0 \% \mathrm{NaCl}$ biofilm cultures,

493 whereas the marine cluster of 13 bacterial taxa had higher relative transcript levels in the

494 Ref300N-23C and 900-30C biofilms cultures. A third cluster showed five bacterial taxa with

495 lower relative transcript levels in the $900 \mathrm{~N}-30 \mathrm{C}$ biofilm cultures compared to the $0 \% \mathrm{NaCl}$ and

496 Ref300N-23C biofilm cultures. In these cases, higher temperature $\left(30^{\circ} \mathrm{C}\right.$ vs $\left.23^{\circ} \mathrm{C}\right)$ and higher

$497 \mathrm{NO}_{3}{ }^{-}$and methanol concentration $\left(64.3 \mathrm{mM}\right.$ vs $21.4 \mathrm{mM} \mathrm{NO}_{3}{ }^{-} ; 0.45 \%$ vs $0.15 \%$ methanol) may

498 have negatively affected these populations.

499

500 Discussion

501 In the environment, numerous bacteria belonging to different taxa can accomplish

502 denitrifying activities, and many of them were encountered in different types of denitrification

503 processes (Lu et al. 2014). Very few studies describing the microbial community of methanol-

504 fed denitrification systems have been reported so far. Most of these studies are based on cloned

505 16S rRNA gene sequences of around 100 clones or based on fluorescence in situ hybridization

506 (Baytshtok et al. 2008; Ginige et al. 2004; Hallin et al. 2006; Neef et al. 1996; Osaka et al. 2008;

507 Osaka et al. 2006; Rissanen et al. 2016; Rissanen et al. 2017; Sun et al. 2016; Yoshie et al.

508 2006). In all these studies, high proportions of Hyphomicrobium spp. were found in combination

509 with high proportions of other methylotrophs such as Methyloversatilis spp., Methylophilus spp.,

510 Methylotenera spp. or Paracoccus spp. The Biodome marine denitrification system showed no

511 exception to this trend with co-occurrence of Hyphomicrobium spp. and the marine methylotroph

512 Methylophaga spp. This co-occurrence was also observed in two other denitrification systems

513 treating saline effluents (Osaka et al. 2006; Rissanen et al. 2016) (see Discussion by Payette et

514 al. (2019). The bacterial diversity of the biofilm taken from the Biodome denitrification system

515 was assessed before when the reactor was operational in 2002 by deriving a 16S rRNA gene

516 library and by culture approach (Labbé et al. 2003; Labbé et al. 2004). Beside Hyphomicrobium

517 sp. and Methylophaga sp., Paracoccus sp., Sulfitobacter sp., Nitratireductor aquibiodomus, and

518 Delftia sp. among others were identified. In the present report, a more complete determination of

519 the composition of the bacterial community of the denitrifying biofilm that was frozen in 2006 
520 (when the denitrification system was dismantle by the Biodome) was possible with the new 521 sequencing technology.

522 The composition of the bacterial community of the OB and the IO biofilm cultures was 523 derived from a different region of the 16S rRNA genes (V6 to V8) than that used for the other 524 biofilm cultures (V1 to V3), and each region was sequenced by a different technology (Illumina 525 and pyrosequencing, respectively). Pyrosequencing technology was no longer available at the 526 time of the sampling of the OB and the IO biofilm cultures, which were carried two years later 527 than the other biofilm cultures. Despites these differences, we believe that the results generated 528 by these two approaches were comparable because they are consistent with the results obtained 529 by qPCR assays (Table 2) that have determined the concentrations of M. nitratireducenticrescens 530 (copies narG1 per ng biofilm DNA) and H. nitrativorans strain NL23 (copies napA per ng 531 biofilm DNA) in the biofilm cultures (Payette et al. 2019). For instance, qPCR showed very low 532 levels of $H$. nitrativorans strain NL23 in the Ref300C-23C biofilm cultures and high level in the 533 OB. These results concur with the absence of $16 \mathrm{~S}$ rRNA sequences associated to

534 Hyphomicrobium spp. in the Ref300C-23C biofilm cultures (pyrosequencing) and high number 535 of these sequences in the OB (Illumina sequencing).

536 The OB cultivated under the different conditions in the ASW medium showed important 537 changes in the Methylophaga and the Hyphomicrobium populations. The proportion of $16 \mathrm{~S}$ 538 rRNA gene sequences associated to Methylophaga spp. was 3.5\% in the OB but was very high, 539 between $85 \%$ and $97 \%$, in these cultures. On the contrary, the proportion of $16 \mathrm{~S}$ rRNA gene 540 sequences associated to Hyphomicrobium spp. was high (46\%) in the OB, but was very low $(0 \%$ 541 to $11 \%$ ) in these cultures. The $\mathrm{NaCl}$ concentrations in the ASW had an impact on the 542 Hyphomicrobium populations. 16S rRNA gene sequences associated to Hyphomicrobium spp. 543 were absent in the biofilm cultures cultivated in ASW at 2.75\% NaCl (Ref300N-23C, 300N-30C, $544900 \mathrm{~N}-23 \mathrm{C}, 900 \mathrm{~N}-30 \mathrm{C}$ ), but were present in the biofilm cultures cultivated at low $\mathrm{NaCl}$ 545 concentrations $(0 \%, 0.5 \%$ and $1 \%)$. These results concur with those obtained by qPCR for the 546 concentrations of M. nitratireducenticrescens and H. nitrativorans strain NL23 (Payette et al. 547 2019). Cultivating the OB in the IO medium had a different impact on the Hyphomicrobium 548 populations. Although the concentrations of salts in the IO medium and in the ASW with $2.75 \%$ $549 \mathrm{NaCl}$ were similar (around 3.5\%), persistence of Hyphomicrobium spp. occurred in the IO 550 biofilm cultures. In fact, the concentrations of $H$. nitrativorans strain NL23 determined by qPCR 
551 between the OB and the IO biofilm cultures was in the same level of magnitude. The lower

552 proportion of $16 \mathrm{~S}$ rRNA gene sequences associated to Hyphomicrobium spp. in the IO biofilm

553 cultures compared to OB was a consequence of the substantial growth of $M$.

554 nitratireducenticrescens in these cultures, with a 10-fold increase in concentration as revealed by

555 qPCR (Table 2) (see Payette et al., 2019 for further discussion). Grob et al. (2015) also observed

556 strong growth of Methylophaga spp. in their seawater samples that were fed with $100 \mu \mathrm{M}$

557 methanol with relative proportions of $16 \mathrm{~S}$ rRNA gene sequences raising from $<0.5 \%$ at $\mathrm{T}=0$ to

$558 \quad 84 \%$ after 3 days.

559 Cultivating the $\mathrm{OB}$ in higher concentrations of $\mathrm{NO}_{3}{ }^{-}$and methanol $(64.3 \mathrm{mM} / 0.45 \%$; $\mathrm{C} / \mathrm{N}$

$5601.5)$ and/or at $30^{\circ} \mathrm{C}(300 \mathrm{~N}-30 \mathrm{C}, 900 \mathrm{~N}-23 \mathrm{C}$ and $900 \mathrm{~N}-30 \mathrm{C}$ biofilm cultures) resulted in increases

561 of $20 \%$ to $85 \%$ in the specific denitrification rates compared to the Ref $300 \mathrm{~N}-23 \mathrm{C}$ biofilm

562 cultures (Table 1) (Payette et al. 2019). Temperature was shown to be the main factor of these

563 increases. However, raising the $\mathrm{NO}_{3}{ }^{-}$and methanol concentrations or/and temperature in these

564 cultures did not have an important impact on the bacterial community when compared to the

565 Ref300N-23C biofilm cultures. Metatranscriptomic analysis of the Ref300N-23C and 900-30C

566 biofilm cultures did not reveal either substantial changes in the gene expression profiles between

567 these two cultures. The higher specific denitrification rates of these biofilm cultures could be

568 related to higher metabolisms at the protein level such as the processing of $\mathrm{NO}_{3}{ }^{-}$by the

569 reductases and transporters.

570 Results from the 16S rRNA gene sequences showed that Marinicella spp. were present in

571 the $\mathrm{OB}$ and all the biofilm cultures, and were the second most abundant bacterial population in

572 the biofilm culture cultivated in $\mathrm{ASW}$ at $2.75 \% \mathrm{NaCl}$. These results concur with the

573 metatranscriptomes of the biofilm cultures where Marinicella spp. had the relative transcript

574 levels among the highest in the de novo assembled transcripts. Marinicella spp. are considered

575 strict aerobic bacteria with no indication of $\mathrm{NO}_{3}{ }^{-}$reduction (Romanenko et al. 2010) although a

576 previous study reported high relative abundances of Marinicella spp. in anoxic sulfide oxidizing

577 reactors in which nitrate was used as the electron acceptor (Huang et al. 2015). Genome

578 annotations of two Marinicella strains (GenBank: Marinicella sp. F2 - assembly number

579 ASM200005v1 and M. litoralis KMM 3900 - ASM259191v1) (Wang et al. 2018) did not reveal

580 complete denitrification pathway, beside a nitric oxide reductase gene cluster also detected here

581 in our metatranscriptomes. Together with the presence of nirS gene (Table 6), these results 
582 indicated that Marinicella spp. might have the capacity to use intermediates of the denitrification 583 cycle to support their growth.

584 The 16S rRNA gene sequences provided evidence of the presence of Pseudomonas spp., 585 Marinobacter spp., Stappia spp., Paracoccus spp. and Aquamicrobium spp. in the OB and in the 586 biofilm cultures. Some species belonging to these genera were reported to carry denitrification.

587 Isolates affiliated to the genera Pseudomonas, Marinobacter and Paracoccus were recovered 588 from the Ref300N-23C biofilm cultures. The Marinobacter and Paracoccus isolates could 589 perform denitrifying activities and grow under anoxic conditions, whereas the Pseudomonas 590 isolate could only consume $\mathrm{NO}_{3}$.

591 Although denitrification genes were found in several of the bacterial populations identified

592 by the metatranscriptomic approach, only transcripts encoding the four denitrification reductases 593 affiliated to Stappia spp. were found in the three examined biofilm cultures. The proportions of 594 Stappia spp. in the 16S rRNA gene sequences of these biofilm cultures ranged between $0.09 \%$ 595 and $0.42 \%$ (Table 2). Stappia spp. are chemoorganotrophic bacteria found in marine 596 environments (Weber \& King 2007) that can oxidize carbon monoxide. They possess the form I 597 coxL gene encoding the large subunit of carbon monoxide (CO) dehydrogenase. Some also 598 contain a gene for the large subunit of ribulose-1,5-bisphosphate carboxylase (RuBisCO) and 599 may be able to couple $\mathrm{CO}$ utilization to $\mathrm{CO}_{2}$ fixation (King 2003). A coxL gene was found in the 600 de novo assembled transcripts affiliated to Stappia spp., but not RuBisCO. Sequences analogue 601 to transporters for simple and multiple sugars such as xylose and fructose, and acetate were 602 found (Supplemental doc 5, 6, 7), which suggest that the Stappia bacteria fed on the biofilm 603 material for carbon sources. Combined with the isolation of denitrifying isolates affiliated to 604 Marinobacter spp. and Paracoccus spp., these results suggest that the biofilm has the potential to 605 adapt to heterotrophic non-methylotrophic environments.

606 The proportion of 16S rRNA gene sequences associated to Bacteroidetes in the OB and in 607 the eight biofilm cultures ranged from $0.2 \%$ to $4.9 \%$, and several genera of this phylum were 608 identified in the three metatranscriptomes. Significant proportions of bacteria affiliated to the 609 Bacteroidetes phylum were also found in other methanol-fed denitrification systems. For 610 instance, $29 \%$ of cloned 16S rRNA gene sequences were affiliated to Bacteroidetes in an 611 acclimated activated sludge in a methanol-fed anoxic denitrification process treating a synthetic 612 wastewater with $4 \% \mathrm{NaCl}$ (Osaka et al. 2008). Isolates affiliated to the Bacteroidetes Aequorivita 
613 spp. and Winogradskyella spp. were isolated from the Ref300N-23C biofilm cultures. None of 614 these two isolates, however, could sustain growth under denitrifying conditions. Although

615 denitrification genes affiliated to Bacteroidetes genera were found in de novo assembled

616 transcripts, genes encoding all four denitrification reductases were not found to any of them.

617 These results suggest that Bacteroidetes are not involved in denitrification, although they may be 618 involved in some steps of the denitrification pathway.

619 The metatranscriptomic data provided some insights of specific metabolisms in $H$.

620 nitrativorans strain NL23 and M. nitratireducenticrescens strain GP59 that were regulated in the

621 biofilm environment. In absence of strain NL23 in the Ref300C-23C and the 900N-30C biofilm

622 cultures, the nirK gene of strain GP59 was upregulated by 5 to 10 times compared to the

623 planktonic pure cultures that was also cultivated under denitrifying conditions. On the contrary,

624 the relative transcript levels of this gene did not change between the $0 \% \mathrm{NaCl}$ biofilm cultures

625 and the planktonic pure cultures, while the relative transcript levels of the NL23 nirK were 49

626 times higher in the $0 \% \mathrm{NaCl}$ biofilm cultures. These results suggest coordination in the

627 expression of nirK between these two strains in the $0 \% \mathrm{NaCl}$ biofilm cultures.

628 The gene clusters encoding the three other denitrification reductases (nap, nor, nos) in

629 strain NL23 showed higher relative transcript levels in the $0 \% \mathrm{NaCl}$ biofilm cultures. napGH

630 was however down regulated in these biofilm cultures. nap $G H$ is located in a separate

631 chromosomic region than the napABCDEF operon. NapGH and NapC have redundant function

632 of transferring electrons to $\mathrm{NapB}$ across the membrane. It was proposed that $\mathrm{NapC}$ transfers

633 electrons from the menaquinol, whereas NapGH do it from ubiquinol (Simon 2011). The

634 physiological consequence of nap $G H$ transcript decrease in the biofilm is unknown.

635 Observations on napEDABC found in the denitrifier Shewanella denitrificans OS217, and 636 napDAGHB in the respiratory $\mathrm{NO}_{3}{ }^{-}$ammonifier Shewanella oneidensis MR-1 suggest that 637 NapGH is more involved in the ammonification system (Simpson et al. 2010). Despite the 638 denitrifying conditions applied in both types of cultures, the biofilm environment has induced 639 strong up regulation of denitrification genes in H. nitrativorans strain NL23. This may be in 640 response to the rapid processing of $\mathrm{NO}_{3}{ }^{-}$by $M$. nitratireducenticrescens strain JAM1/GP59

641 (Mauffrey et al. 2015) that could generate rapidly high level of $\mathrm{NO}_{2}^{-}$, which is toxic for strain $642 \quad$ NL23.

643 


\section{Conclusion}

645 The OB taken from the Biodome denitrification system underwent substantial changes in 646 its bacterial community when subjected to environmental changes. Cultivating the OB in the 647 homemade ASW medium with different formulations (varying $\mathrm{NaCl}, \mathrm{NO}_{3}{ }^{-}$and methanol 648 concentrations, and temperature) or in the commercial IO medium showed much higher 649 proportions of Methylophaga spp. in these biofilm cultures compared to the OB. These results 650 concur with the growth of M. nitratireducenticrescens strain GP59 in these cultures. The 651 population of Hyphomicrobium spp showed a more complex trend. It was not detected in the 652 biofilm cultures cultivated in $\mathrm{ASW}$ at $2.75 \% \mathrm{NaCl}$, but persisted in the biofilm cultures 653 cultivated in $\mathrm{ASW}$ at low $\mathrm{NaCl}$ concentration, and also cultivated in the IO medium. Other 654 denitrifiers affiliated to Marinobacter spp. and Paracoccus spp. were isolated from the biofilm 655 cultures. Moreover, metatranscriptomic analysis revealed that denitrifying bacteria affiliated to 656 Stappia spp. were metabolically active in the biofilm cultures. The biofilm environment has 657 favored the upregulation of the denitrification pathway in M. nitratireducenticrescens strain 658 GP59 and H. nitrativorans strain NL23 compared to planktonic pure cultures, despite the facts 659 that these two types of cultures were grown under denitrifying conditions. All these results 660 demonstrated the dynamics and the plasticity of the denitrifying biofilm to sustain environmental 661 changes and illustrate a comprehensive picture of the microbial community of the biofilm and its 662 adaptation to these changes. This could benefit in the development of optimal denitrifying 663 bioprocess under marine conditions. For instance, the fact that non-methylotrophic, denitrifying 664 bacteria are present in the biofilm could suggest adaptation of denitrification process to another 665 source of carbon such as ethanol or acetate.

666

667 Acknowledgements

668 We thank Karla Vasquez for her technical assistance.

669

670

\section{References}

671

672

Atlas RM. 1993. Handbook of microbiological media. Ed. Parks L. C. CRC press Boca Raton, 673 Fl, USA. pp 543. 
674 Auclair J, Lepine F, Parent S, and Villemur R. 2010. Dissimilatory reduction of nitrate in 675 seawater by a Methylophaga strain containing two highly divergent narG sequences. ISME Journal 4:1302-1313. doi: 10.1038/ismej.2010.47

677

678

679

680

681

682

683

684

685

686

687

688

689

690

691

692

693

694

695

696

697

698

699

700

701

702

703

704

Auclair J, Parent S, and Villemur R. 2012. Functional diversity in the denitrifying biofilm of the methanol-fed marine denitrification system at the Montreal Biodome. Microb Ecol 63:726-735. doi: 10.1007/s00248-011-9960-2

Baytshtok V, Kim S, Yu R, Park H, and Chandran K. 2008. Molecular and biokinetic characterization of methylotrophic denitrification using nitrate and nitrite as terminal electron acceptors. Water Science and Technology 58:359-365. doi: 10.2166/wst.2008.391

Cabrol L, and Malhautier L. 2011. Integrating microbial ecology in bioprocess understanding: the case of gas biofiltration. Appl Microbiol Biotechnol 90:837-849. doi: 10.1007/s00253-011-3191-9

Chistoserdova L, Chen SW, Lapidus A, and Lidstrom ME. 2003. Methylotrophy in Methylobacterium extorquens AM1 from a genomic point of view. J Bacteriol 185:29802987. doi: $10.1128 / \mathrm{Jb} .185 .10 .2980-2987.2003$

Chistoserdova L, Laukel M, Portais JC, Vorholt JA, and Lidstrom ME. 2004. Multiple formate dehydrogenase enzymes in the facultative methylotroph Methylobacterium extorquens AM1 are dispensable for growth on methanol. $J$ Bacteriol 186:22-28.

Clark ME, He ZL, Redding AM, Joachimiak MP, Keasling JD, Zhou JZZ, Arkin AP, Mukhopadhyay A, and Fields MW. 2012. Transcriptomic and proteomic analyses of Desulfovibrio vulgaris biofilms: Carbon and energy flow contribute to the distinct biofilm growth state. Bmc Genomics 13. doi: 10.1186/1471-2164-13-138

Cole JR, Wang Q, Fish JA, Chai B, McGarrell DM, Sun Y, Brown CT, Porras-Alfaro A, Kuske CR, and Tiedje JM. 2014. Ribosomal Database Project: data and tools for high throughput rRNA analysis. Nucleic Acids Res 42:D633-642. doi: 10.1093/nar/gkt1244

Edgar RC. 2013. UPARSE: highly accurate OTU sequences from microbial amplicon reads. Nat Methods 10:996-+. doi: 10.1038/Nmeth.2604

Edgar RC, Haas BJ, Clemente JC, Quince C, and Knight R. 2011. UCHIME improves sensitivity and speed of chimera detection. Bioinformatics 27:2194-2200. doi: 10.1093/bioinformatics/btr381

Peer) reviewing PDF | (2019:04:36595:2:0:ACCEPTED 10 Jul 2019) 
705 Geoffroy V, Payette G, Mauffrey F, Lestin L, Constant P, and Villemur R. 2018. Strain-level

706

707

708

709

710

711

712

713

714

715

716

717

718

719

720

721

722

723

724

725

726

727

728

729

730

731

732

733 genetic diversity of Methylophaga nitratireducenticrescens confers plasticity to denitrification capacity in a methylotrophic marine denitrifying biofilm. PeerJ 6:e4679. doi: $10.7717 /$ peerj.4679

Ginige MP, Hugenholtz P, Daims H, Wagner M, Keller J, and Blackall LL. 2004. Use of stableisotope probing, full-cycle rRNA analysis, and fluorescence in situ hybridizationmicroautoradiography to study a methanol-fed denitrifying microbial community. Appl Environ Microbiol 70:588-596. doi: 10.1128/Aem.70.1.588-596.2004

Grabherr MG, Haas BJ, Yassour M, Levin JZ, Thompson DA, Amit I, Adiconis X, Fan L, Raychowdhury R, Zeng Q, Chen Z, Mauceli E, Hacohen N, Gnirke A, Rhind N, di Palma F, Birren BW, Nusbaum C, Lindblad-Toh K, Friedman N, and Regev A. 2011. Fulllength transcriptome assembly from RNA-Seq data without a reference genome. Nat Biotechnol 29:644-652. doi: 10.1038/nbt.1883

Grob C, Taubert M, Howat AM, Burns OJ, Dixon JL, Richnow HH, Jehmlich N, von Bergen M, Chen Y, and Murrell JC. 2015. Combining metagenomics with metaproteomics and stable isotope probing reveals metabolic pathways used by a naturally occurring marine methylotroph. Environ Microbiol 17:4007-4018. doi: 10.1111/1462-2920.12935

Hallin S, Throback IN, Dicksved J, and Pell M. 2006. Metabolic profiles and genetic diversity of denitrifying communities in activated sludge after addition of methanol or ethanol. Appl Environ Microbiol 72:5445-5452. doi: 10.1128/AEM.00809-06

Huang C, Zhao YK, Li ZL, Yuan Y, Chen C, Tan WB, Gao S, Gao LF, Zhou JZ, and Wang AJ. 2015. Enhanced elementary sulfur recovery with sequential sulfate-reducing, denitrifying sulfide-oxidizing processes in a cylindrical-type anaerobic baffled reactor. Bioresource Technology 192:478-485. doi: 10.1016/j.biortech.2015.04.103

King GM. 2003. Molecular and culture-based analyses of aerobic carbon monoxide oxidizer diversity. Appl Environ Microbiol 69:7257-7265.

Kraft B, Strous M, and Tegetmeyer HE. 2011. Microbial nitrate respiration - Genes, enzymes and environmental distribution. Journal of Biotechnology 155:104-117. doi: 10.1016/j.jbiotec.2010.12.025 
734 Labbé N, Juteau P, Parent S, and Villemur R. 2003. Bacterial diversity in a marine methanol-fed

735

736

737

738

739

740

741

742

743

744

745

746

747

748

749

750

751

752

753

754

755

756

757

758

759

760

761

762

763 denitrification reactor at the Montreal Biodome, Canada. Microb Ecol 46:12-21. doi: $10.1007 / \mathrm{s} 00248-002-1056-6$

Labbé N, Laurin V, Juteau P, Parent S, and Villemur R. 2007. Microbiological community structure of the biofilm of a methanol-fed, marine denitrification system, and identification of the methanol-utilizing microorganisms. Microb Ecol 53:621-630. doi: $10.1007 / \mathrm{s} 00248-006-9168-\mathrm{Z}$

Labbé N, Parent S, and Villemur R. 2004. Nitratireductor aquibiodomus gen. nov., sp nov., a novel alpha-proteobacterium from the marine denitrification system of the Montreal Biodome (Canada). International Journal of Systematic and Evolutionary Microbiology 54:269-273. doi: 10.1099/ijs.0.02793-0

Li B, and Dewey CN. 2011. RSEM: accurate transcript quantification from RNA-Seq data with or without a reference genome. BMC Bioinformatics 12:323. doi: 10.1186/1471-2105-12323

Lu HJ, Chandran K, and Stensel D. 2014. Microbial ecology of denitrification in biological wastewater treatment. Water Research 64:237-254. doi: 10.1016/j.waters.2014.06.42

Martineau C, Mauffrey F, and Villemur R. 2015. Comparative analysis of denitrifying activities of Hyphomicrobium nitrativorans, Hyphomicrobium denitrificans, and Hyphomicrobium zavarzinii. Appl Environ Microbiol 81:5003-5014. doi: 10.1128/Aem.00848-15

Martineau C, Villeneuve C, Mauffrey F, and Villemur R. 2013a. Complete genome sequence of Hyphomicrobium nitrativorans strain NL23, a denitrifying bacterium isolated from biofilm of a methanol-fed denitrification system treating seawater at the Montreal Biodome. Genome Announc 2: e01165-13. doi: 10.1128/genomeA.01165-13

Martineau C, Villeneuve C, Mauffrey F, and Villemur R. 2013b. Hyphomicrobium nitrativorans sp. nov., isolated from the biofilm of a methanol-fed denitrification system treating seawater at the Montreal Biodome. Int J Syst Evol Microbiol 63:3777-3781. doi: 10.1099/ijs.0.048124-0

Mauffrey F, Cucaita A, Constant P, and Villemur R. 2017. Denitrifying metabolism of the methylotrophic marine bacterium Methylophaga nitratireducenticrescens strain JAM1. Peerj 5:e4098. doi: 10.7717/Peerj.4098 
764 Mauffrey F, Martineau C, and Villemur R. 2015. Importance of the two dissimilatory (Nar)

765

766

767

768

769

770

771

772

773

774

775

776

777

778

779

780

781

782

783

784

785

786

787

788

789

790

791

792

793

794

nitrate reductases in the growth and nitrate reduction of the methylotrophic marine bacterium Methylophaga nitratireducenticrescens JAM1. Front Microbiol 6:1475.

Metsalu T, and Vilo J. 2015. ClustVis: a web tool for visualizing clustering of multivariate data using Principal Component Analysis and heatmap. Nucleic Acids Res 43:W566-570. doi: 10.1093/nar/gkv468

Neef A, Zaglauer A, Meier H, Amann R, Lemmer H, and Schleifer KH. 1996. Population analysis in a denitrifying sand filter: Conventional and in situ identification of Paracoccus spp. in methanol-fed biofilms. Appl Environ Microbiol 62:4329-4339.

Osaka T, Shirotani K, Yoshie S, and Tsuneda S. 2008. Effects of carbon source on denitrification efficiency and microbial community structure in a saline wastewater treatment process. Water Research 42:3709-3718. doi: 10.1016/j.watres.2008.06.007

Osaka T, Yoshie S, Tsuneda S, Hirata A, Iwami N, and Inamori Y. 2006. Identification of acetate- or methanol-assimilating bacteria under nitrate-reducing conditions by stableisotope probing. Microb Ecology 52:253-266. doi: 10.1007/s00248-006-9071-7

Payette G, Geoffroy V, Martineau C, and Villemur R. 2019. Dynamics of a methanol-fed marine denitrifying biofilm: 1-Impact of environmental changes on the denitrification and the cooccurrence of Methylophaga nitratireducenticrescens and Hyphomicrobium nitrativorans. PeerJ submitted.

Peck V, Quiza L, Buffet JP, Khdhiri M, Durand AA, Paquette A, Thiffault N, Messier C, Beaulieu N, Guertin C, and Constant P. 2016. Towards the development of multifunctional molecular indicators combining soil biogeochemical and microbiological variables to predict the ecological integrity of silvicultural practices. Microbial Biotechnology 9:316-329. doi: 10.1111/1751-7915.12348

Peyraud R, Kiefer P, Christen P, Massou S, Portais JC, and Vorholt JA. 2009. Demonstration of the ethylmalonyl-CoA pathway by using C-13 metabolomics. Proceedings of the National Academy of Sciences of the United States of America 106:4846-4851. doi: 10.1073/pnas.0810932106

Philippot L, and Hojberg O. 1999. Dissimilatory nitrate reductases in bacteria. Biochimica Et Biophysica Acta-Gene Structure and Expression 1446:1-23. doi: 10.1016/S01674781(99)00072-X

Peer] reviewing PDF | (2019:04:36595:2:0:ACCEPTED 10 Jul 2019) 
795 Resch A, Rosenstein R, Nerz C, and Gotz F. 2005. Differential gene expression profiling of

796

797

798

799

800

801

802

803

804

805

806

807

808

809

810

811

812

813

814

815

816

817

818

819

820

821

822

823

824

825

Staphylococcus aureus cultivated under biofilm and planktonic conditions. Appl Environ Microbiol 71:2663-2676. doi: 10.1128/Aem.71.5.2663-2676.2005

Richardson D, Berks B, Russell D, Spiro S, and Taylor C. 2001. Functional, biochemical and genetic diversity of prokaryotic nitrate reductases. Cellular and Molecular Life Sciences CMLS 58:165-178.

Rissanen AJ, Ojala A, Dernjatin M, Jaakkola J, and Tiirola M. 2016. Methylophaga and Hyphomicrobium can be used as target genera in monitoring saline water methanolutilizing denitrification. Journal of Industrial Microbiology and Biotechnology 43:16471657. doi: 10.1007/s10295-016-1839-2

Rissanen AJ, Ojala A, Fred T, Toivonen J, and Tiirola M. 2017. Methylophilaceae and Hyphomicrobium as target taxonomic groups in monitoring the function of methanol-fed denitrification biofilters in municipal wastewater treatment plants. Journal of Industrial Microbiology and Biotechnology 44:35-47. doi: 10.1007/s10295-016-1860-5

Roder HL, Sorensen SJ, and Burmolle M. 2016. Studying Bacterial Multispecies Biofilms: Where to Start? Trends Microbiol 24:503-513. doi: 10.1016/j.tim.2016.02.019

Romanenko LA, Tanaka N, Frolova GM, and Mikhailov VV. 2010. Marinicella litoralis gen. nov., sp. nov., a gammaproteobacterium isolated from coastal seawater. Int J Syst Evol Microbiol 60:1613-1619. doi: 10.1099/ijs.0.016147-0

Salta M, Wharton JA, Blache Y, Stokes KR, and Briand JF. 2013. Marine biofilms on artificial surfaces: structure and dynamics. Environ Microbiol 15:2879-2893. doi: 10.1111/14622920.12186

Simon J. 2011. Organization of respiratory electron transport chains in nitrate-reducing and nitrifying bacteria. In: Moir JWB, ed. Nitrogen cycling in bacteria Molecular analysis Caister academic press, Norfolk, UK. pp 39-58.

Simpson PJ, Richardson DJ, and Codd R. 2010. The periplasmic nitrate reductase in Shewanella: the resolution, distribution and functional implications of two NAP isoforms, NapEDABC and NapDAGHB. Microbiology 156:302-312. doi: 10.1099/mic.0.034421-0

Sun Y, Shen D, Zhou X, Shi N, and Tian Y. 2016. Microbial diversity and community structure of denitrifying biological filters operated with different carbon sources. Springerplus 5:1752. doi: 10.1186/s40064-016-3451-3 
826 Tan CH, Lee KW, Burmolle M, Kjelleberg S, and Rice SA. 2017. All together now:

827 experimental multispecies biofilm model systems. Environ Microbiol 19:42-53. doi:

$828 \quad 10.1111 / 1462-2920.13594$

829 Tarazona S, Garcia-Alcalde F, Dopazo J, Ferrer A, and Conesa A. 2011. Differential expression

830 in RNA-seq: A matter of depth. Genome Research 21:2213-2223. doi:

$831 \quad 10.1101 /$ gr. 124321.111

832 Team RC. 2015. A language and environment for statistical computing. R Foundation for 833 Statistical Computing, Vienna, Austria. URL https://www.R-project.org/.

834 Villeneuve C, Martineau C, Mauffrey F, and Villemur R. 2012. Complete genome sequences of 835 Methylophaga sp. strain JAM1 and Methylophaga sp. strain JAM7. J Bacteriol 194:4126836 4127. doi: $10.1128 / \mathrm{Jb} .00726-12$

837 Villeneuve C, Martineau C, Mauffrey F, and Villemur R. 2013. Methylophaga 838 nitratireducenticrescens sp. nov. and Methylophaga frappieri sp. nov., isolated from the biofilm of the methanol-fed denitrification system treating the seawater at the Montreal

841 Wang XQ, Li CM, Dunlap CA, Rooney AP, and Du ZJ. 2018. Marinicella sediminis sp nov., 842 isolated from marine sediment. International Journal of Systematic and Evolutionary Microbiology 68:2335-2339. doi: 10.1099/ijsem.0.002839

844 Weber CF, and King GM. 2007. Physiological, ecological, and phylogenetic characterization of Stappia, a marine CO-oxidizing bacterial genus. Appl Environ Microbiol 73:1266-1276. doi: 10.1128/AEM.01724-06

848

Wright ES, Yilmaz LS, and Noguera DR. 2012. DECIPHER, a search-based approach to chimera identification for 16S rRNA sequences. Appl Environ Microbiol 78:717-725. doi: 10.1128/AEM.06516-11

Yoshie S, Ogawa T, Makino H, Hirosawa H, Tsuneda S, and Hirata A. 2006. Characteristics of 851

852 bacteria showing high denitrification activity in saline wastewater. Lett Appl Microbiol 42:277-283. doi: 10.1111/j.1472-765X.2005.01839.x

853

854 
Figure 1

Proportion of affiliated OTUs in the biofilm cultures

Panel A. Bacterial composition of $\mathrm{OB}$ and the $\mathrm{IO}$ biofilm cultures was determined by sequencing the V6-V7-V8 variable regions of the 16S rRNA gene by Illumina, whereas the other samples were determined by sequencing the $\mathrm{V} 1-\mathrm{V} 2-\mathrm{V} 3$ variable regions by pyrosequencing. Panel B. Principal component analysis of the bacterial profiles of the biofilm cultures and the OB listed in Table 2. 

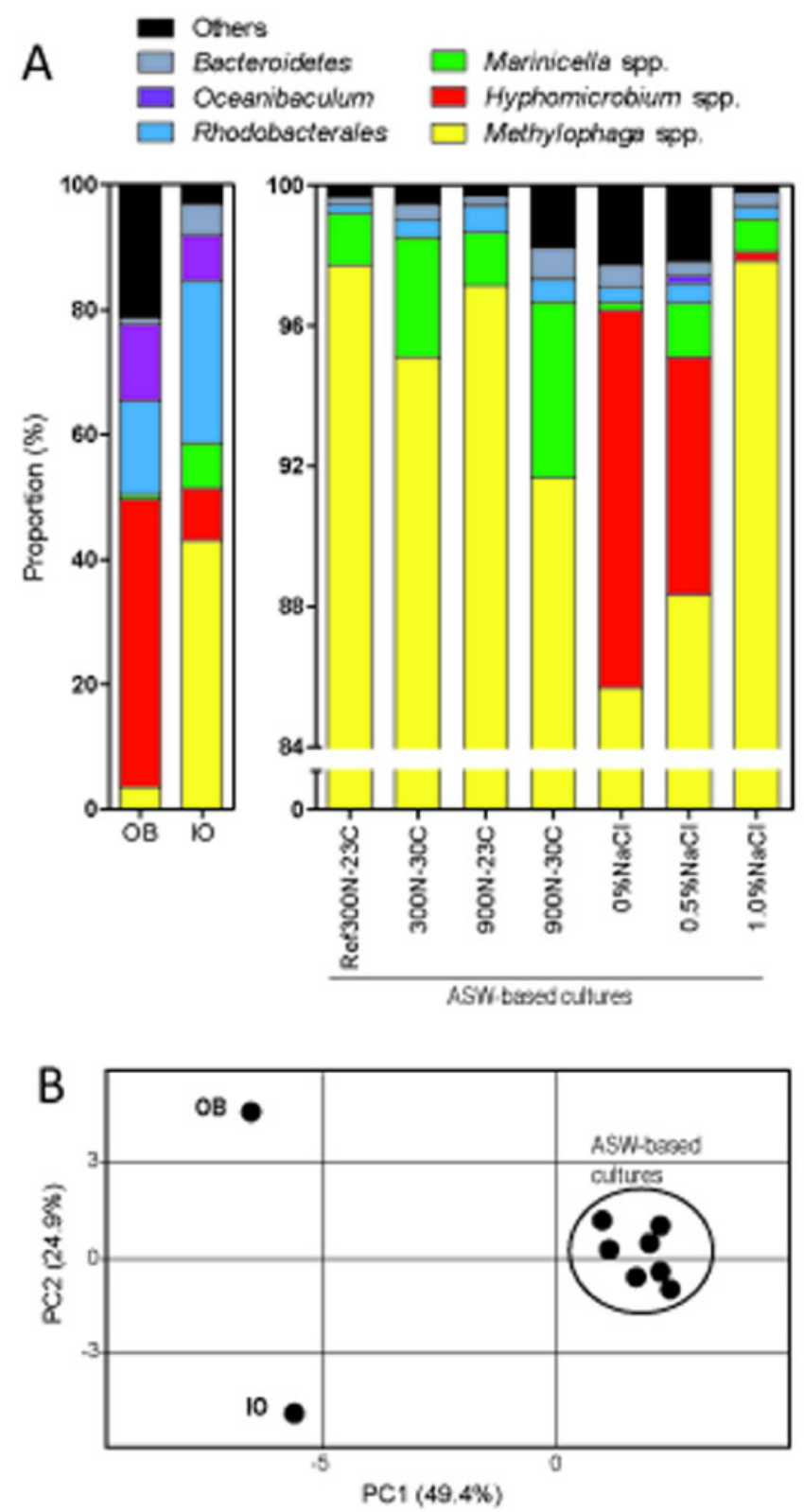


\section{Figure 2}

Relative expression profiles of $M$. nitratireducenticrescens GP59 and $H$. nitrativorans NL23 in biofilm cultures

All the deduced amino acid sequences associated to the genome and plasmids of strain GP59 and the genome of strain NL23 were submitted to the BlastKOALA (genome annotation and KEGG mapping) at the Kyoto encyclopedia of genes and genomes (KEGG). Genes associated to specific metabolisms were sorted out and the corresponding ratio of the Biofilm Transcripts Per Million (TPM) versus the pure culture TPM was derived. When the ratios were $<1$, the negative inverse value $(-1 /$ ratio) was calculated. Data are expressed as the percentage of genes in each category that are more expressed in the biofilm cultures (right, 2 to 5 times, and $>5$ times) or in pure cultures (left, -2 to -5 times and $>-5$ times). Number within parentheses is the number of genes involved in the selected pathways. Other metabolic profiles are detailed in Figures S3 and S4. 


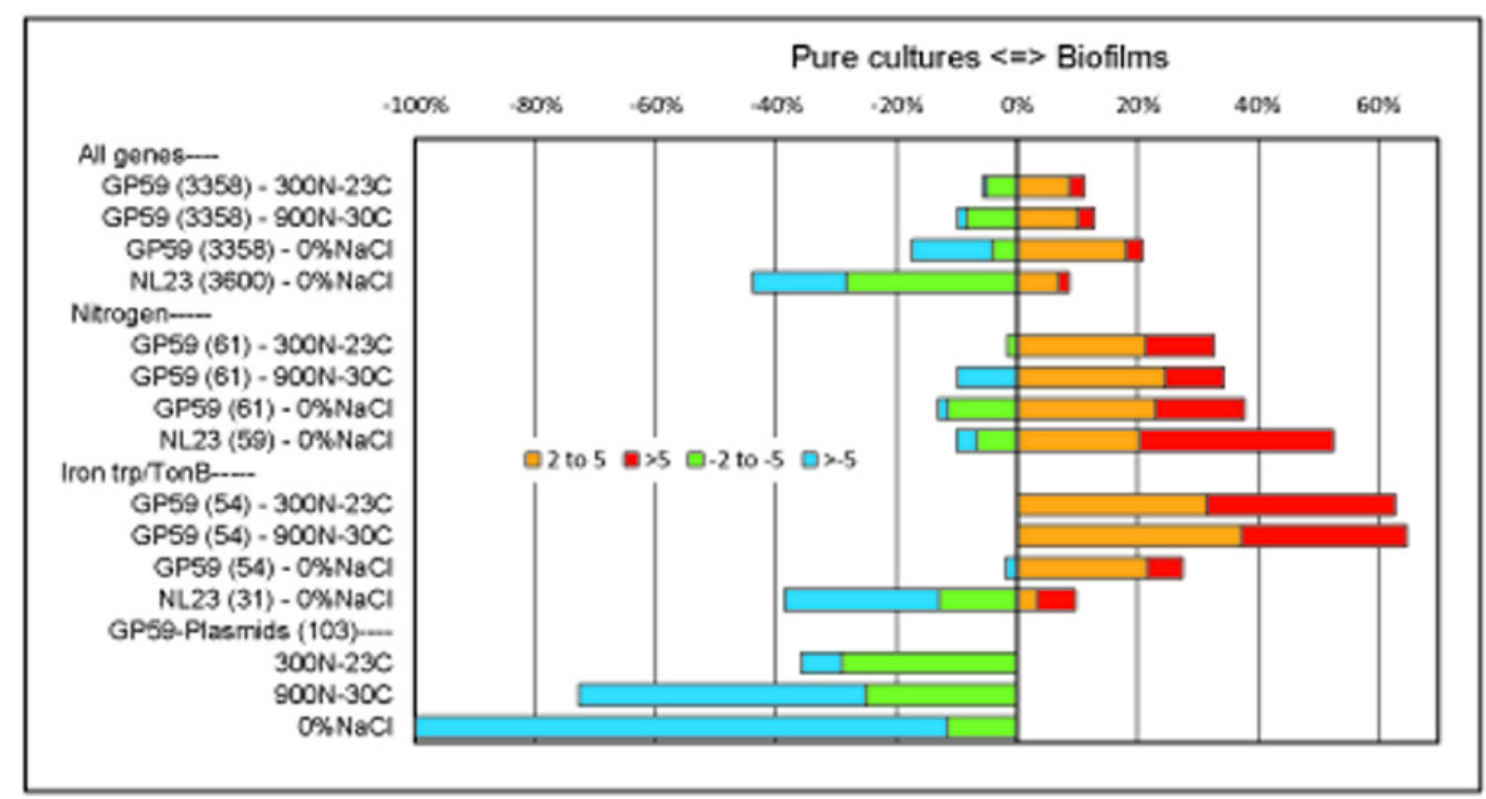




\section{Figure 3}

Relative gene expression profiles of selected metabolic pathways of $M$. nitratireducenticrescens GP59 in the biofilm cultures

The pathways are based on functions deduced by the annotations (provided by KEGG BlastKoala, RAST and GenBank). The arrow thickness is proportional to the value of the ratio of the Biofilm TPM divided by the planktonic pure-culture TPM. The blue arrows represent genes with at least 2-fold lower relative transcript levels in the biofilm cultures. The red arrows represent genes with at least 2 -fold higher relative transcript levels in the biofilm cultures. The black arrows represent no changes between both types of cultures in the relative transcript levels. The two-component systems and the transporters that are illustrated in blue are encoded by strains GP59 and NL23. See Supplemental doc 9 and 10 for gene description. 


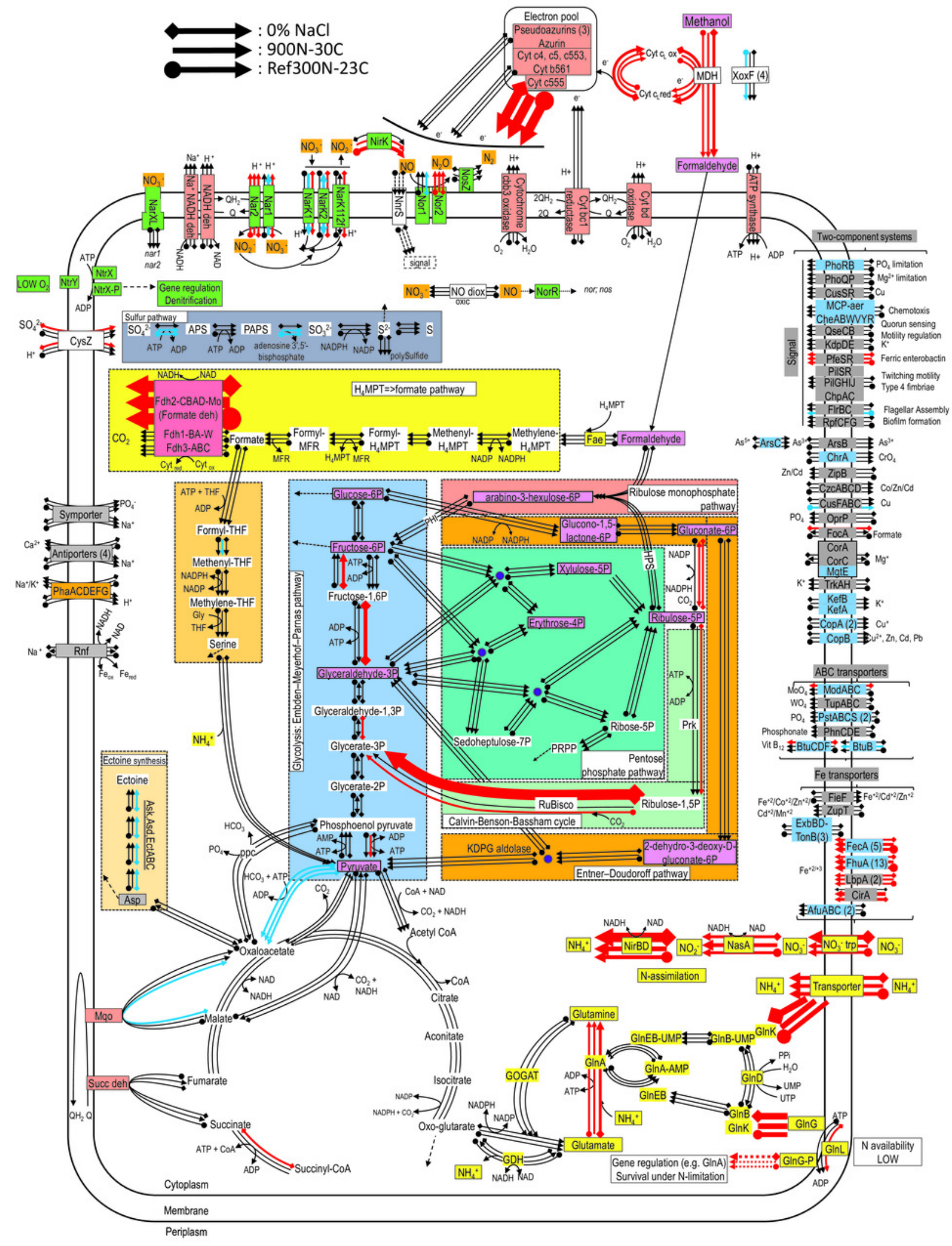


Figure 4

Relative gene expression profiles of selected metabolic pathways of $H$. nitrativorans strain NL23 in the $0 \% \mathrm{NaCl}$ biofilm cultures

See Figure 3 legend. 


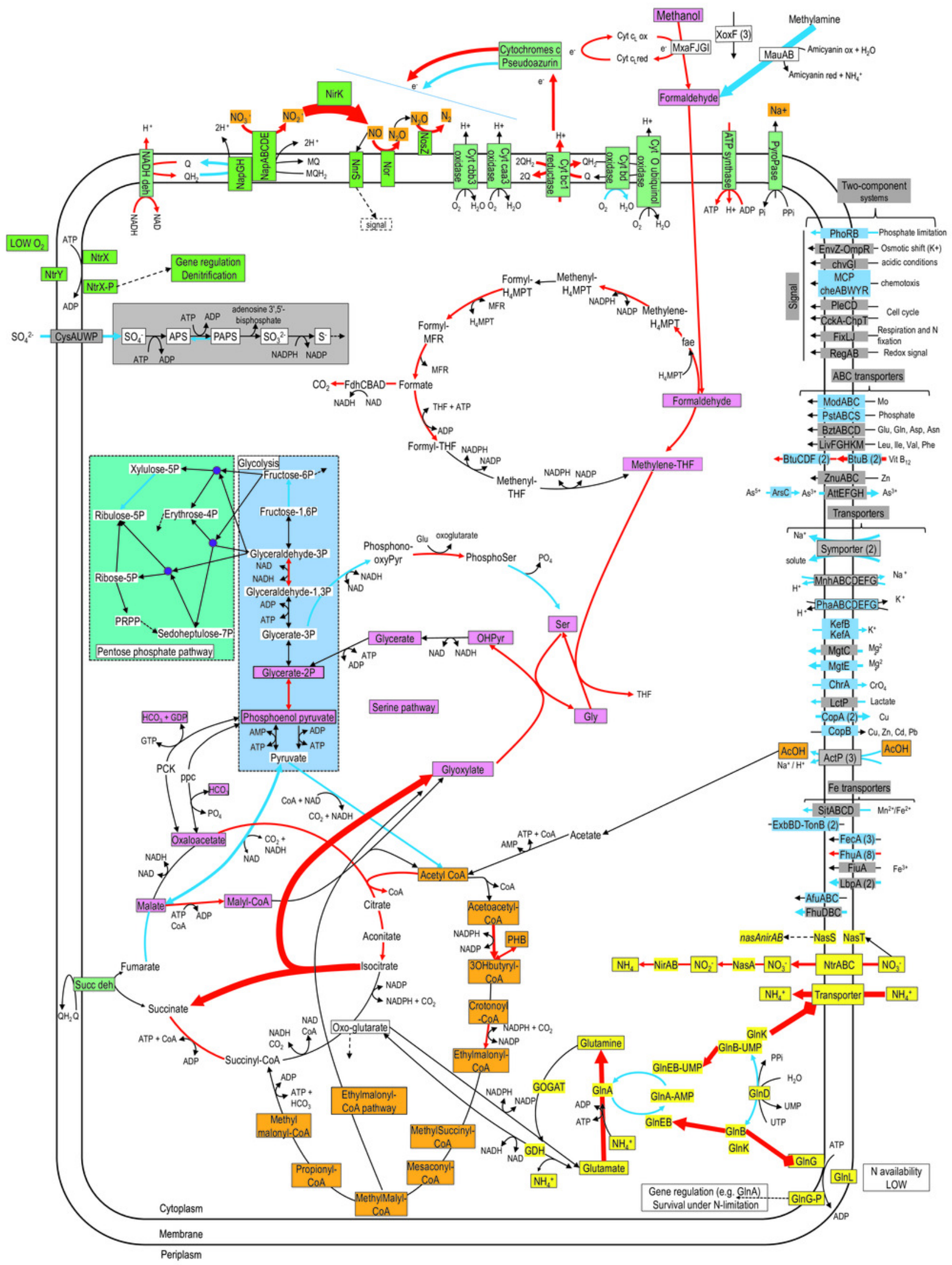




\section{Figure 5}

Hierarchical clustering of the selected bacterial taxa in biofilm culture metatranscriptomes

Heatmap represents differences in the overall gene expression patterns (expressed as TPM; from Table 6) $\left(\log _{10}\right.$ [TPM by geometric average of TPM]) between the three biofilm cultures for the respective bacterial taxa. Analysis was performed at ClustVis web site (https://biit.cs.ut.ee/clustvis/) ( Metsalu and Vilo, 2015 ) . 


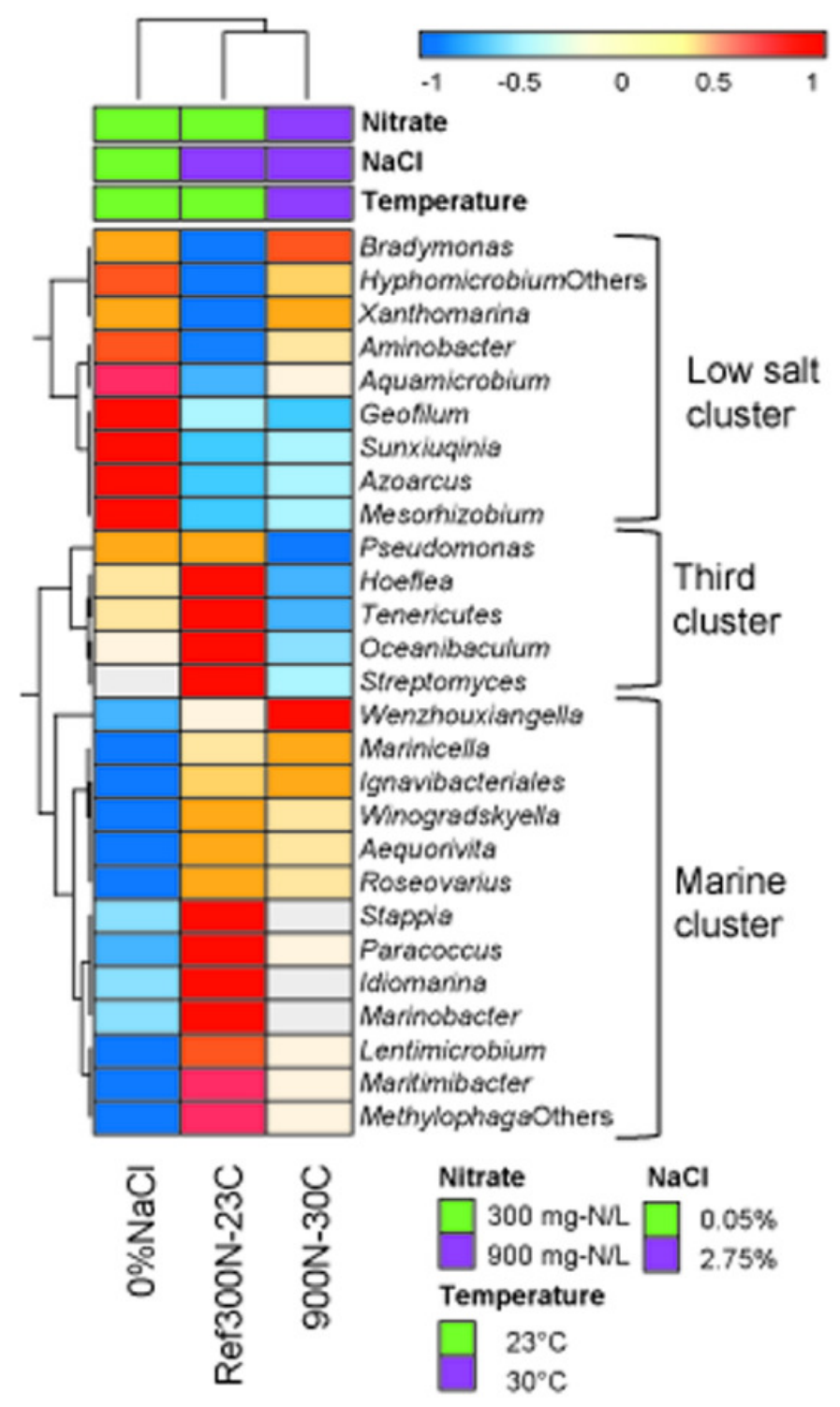


Table $\mathbf{1}$ (on next page)

Biofilm culture conditions 


\section{Table 1. Biofilm culture conditions}

2

3

4

5

6

7

19 a From Payette et al. (2019).

$20{ }^{\mathrm{b}}$ Reference biofilm cultures.

${ }^{c}$ The $\mathrm{C} / \mathrm{N}$ ratio was 1.5 in all biofilm cultures

\begin{tabular}{|c|c|c|c|c|c|c|}
\hline Name & Medium & $\mathrm{NO}_{3}{ }^{-}$ & Methano & $\mathrm{NaCl}$ & Temp & $\begin{array}{l}\text { Specific a } \\
\text { denitrification } \\
\text { rates }\end{array}$ \\
\hline & & $\begin{array}{l}\mathrm{mM} \\
\left(\mathrm{mg}-\mathrm{NO}_{3}-\mathrm{N} / \mathrm{L}\right)^{\mathrm{c}}\end{array}$ & $\%(\mathrm{v} / \mathrm{v})^{\mathrm{c}}$ & $\%(\mathrm{w} / \mathrm{v})$ & ${ }^{\circ} \mathrm{C}$ & $\begin{array}{l}\text { mM-NO } \mathrm{h}^{-1} \\
\text { mg-protein }^{-1}\end{array}$ \\
\hline $\operatorname{Ref} 300 \mathrm{~N}-23 \mathrm{C}^{\mathrm{b}}$ & ASW & $21.4(300)$ & 0.15 & 2.75 & 23 & 0.0530 \\
\hline $300 \mathrm{~N}-30 \mathrm{C}$ & ASW & $21.4(300)$ & 0.15 & 2.75 & 30 & 0.0946 \\
\hline $900 \mathrm{~N}-23 \mathrm{C}$ & ASW & $64.3(900)$ & 0.45 & 2.75 & 23 & 0.0637 \\
\hline $900 \mathrm{~N}-30 \mathrm{C}$ & ASW & $64.3(900)$ & 0.45 & 2.75 & 30 & 0.0979 \\
\hline$\% \mathrm{NaCl}$ & ASW & $21.4(300)$ & 0.15 & 0 & 23 & 0.0911 \\
\hline $0.5 \% \mathrm{NaCl}$ & ASW & $21.4(300)$ & 0.15 & 0.5 & 23 & 0.0712 \\
\hline $1 \% \mathrm{NaCl}$ & ASW & $21.4(300)$ & 0.15 & 1.0 & 23 & 0.0357 \\
\hline [O & IO & $21.4(300)$ & 0.15 & $3.0^{\mathrm{d}}$ & 23 & 0.0611 \\
\hline
\end{tabular}

The original biofilm was cultured in triplicates in these conditions at $\mathrm{pH} 8.0$. The carriers were transferred 5 times in fresh medium around each week before measuring the denitrifying activities.

${ }^{d}$ The exact amount of $\mathrm{NaCl}$ added in the IO medium is not known. See Payette et al. (2019) for the IO composition. For comparison, the amount of $\mathrm{Na}^{+}$and $\mathrm{Cl}^{-}$in the ASW medium is $3.2 \%$. In gray are changed parameters from the reference biofilm cultures.

IO: Instant Ocean medium. 


\section{Table 2 (on next page)}

Most probable affiliation of $16 \mathrm{~S}$ rRNA gene sequences in the biofilm cultures and the original biofilm 
Table 2. Most probable affiliation of $16 \mathrm{~S}$ rRNA gene sequences in the biofilm cultures and the original biofilm

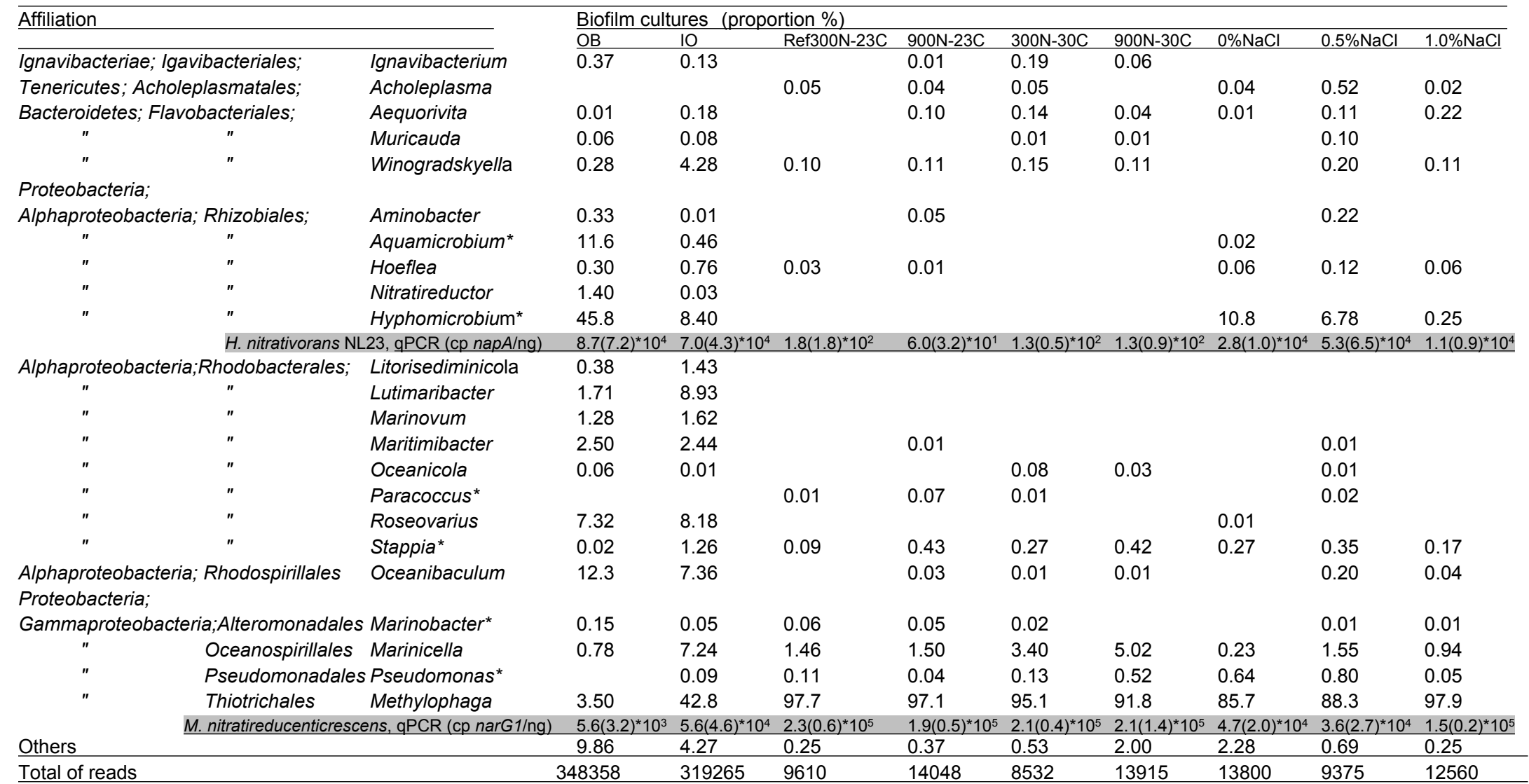

The V1-V3 regions of the 16S rRNA gene sequences were amplified and sequenced by pyrosequencing except for the OB and IO samples from which the V6-V8 regions were sequenced by Illumina. (see supplemental document 2 for complete analysis and sequences). OB: Original biofilm.

In grey: qPCR results are from Payette et al. (2019), with standard deviation values under parentheses of triplicate cultures. narG1 used in qPCR for M. nitratireducenticrescens cannot discriminate strain JAM1 and strain GP59 (identical sequences between the two).

* Identified genus with species that were reported involved in denitrification. 


\section{Table 3(on next page)}

16S Operational Taxonomic Units (OTUs) affiliated to Hyphomicrobium spp. and Methylophaga spp. in the $\mathrm{OB}$ and the IO biofilm cultures 
1 Table 3: 16S Operational Taxonomic Units (OTUs) affiliated to Hyphomicrobium spp. and 2 Methylophaga spp. in the $\mathrm{OB}$ and the IO biofilm cultures

3

4

5

6

\begin{tabular}{|c|c|c|c|c|c|c|}
\hline & \multirow{2}{*}{$\begin{array}{l}\text { Sequence identity with } \\
\text { H. nitrativorans }\end{array}$} & \multirow{2}{*}{$\begin{array}{l}\text { Number of } \\
\text { OTUs }\end{array}$} & \multicolumn{2}{|c|}{ Number reads } & \multicolumn{2}{|c|}{ Proportion of reads $\%$} \\
\hline & & & OB & IO & OB & $\mathrm{IO}$ \\
\hline Cluster 1 & $95-100 \%$ & 12 & 147209 & 26239 & 92.3 & 97.8 \\
\hline Cluster 2 & $91-97 \%$ & 5 & 10779 & 530 & 6.8 & 2.0 \\
\hline Cluster 3 & $89-92 \%$ & 3 & 1537 & 51 & 0.96 & 0.19 \\
\hline & $\begin{array}{l}\text { Sequence identity with } \\
\text { M. nitratireducenticrescens }\end{array}$ & & & & & \\
\hline Cluster 1 & $94-100 \%$ & 18 & 11809 & 136197 & 96.4 & 99.7 \\
\hline Cluster 2 & $92.9-93.6 \%$ & 3 & 321 & 284 & 2.62 & 0.21 \\
\hline Cluster 3 & $93.4-94.1 \%$ & 3 & 115 & 65 & 0.94 & 0.05 \\
\hline
\end{tabular}

Cluster classification is based on phylogenic analyses of the 16S rRNA gene sequences affiliated to $H$. nitrativorans and M. nitratireducenticrescens (see Tables S1 and S2, and Figures S2A and S2b). 


\section{Table 4 (on next page)}

Affiliation of the isolates isolated from the reference biofilm cultures (Ref300N-23C) 


\begin{tabular}{ll}
$\begin{array}{l}\text { Table 4. Affiliation of the isolates isolated from the } \\
\text { reference biofilm cultures (Ref300N-23C) }\end{array}$ \\
\hline Isolates & $\begin{array}{l}\text { Denitrifying } \\
\text { activities* }\end{array}$ \\
\hline Alphaproteobacteria, Rhodobacterales & no \\
Rhodobacteraceae GP11 & Full \\
Paracoccus sp. GP3, GP8, GP20 & no \\
Roseovarius sp. GP9, GP10, GP13, GP14 & no \\
Roseovarius sp. GP12 & no \\
Thalassobius sp. GP19 & \\
Gammaproteobacteria & Full \\
Marinobacter sp. GP1, GP2, GP24 & Nitrate \\
Pseudomonas GP41 & \\
Bacteroidetes, Flavobacteriales & no \\
Aequorivita sp. GP15 & no \\
Winogradskyella sp. GP16, GP18 & \\
Bacillales & no \\
Exiguobacterium sp. GP46
\end{tabular}

* Full: nitrate and nitrite are consumed. Nitrate: only nitrate was consumed 


\section{Table 5 (on next page)}

Changes in the relative transcript levels of genes involved in the nitrogen metabolism in strain GP59 and strain NL23 
1 Table 5: Changes in the relative transcript levels of genes involved in the nitrogen metabolism in strain GP59 and strain NL23

3

4

5

6

7

8

9

10

11

12

13

14

15

16

17

18

19

20

21

22

23

24

25

26

27

28

29

30

31

32

33

34

35

36

37

38

39

40

41

42

43

44

45

46

47

\begin{tabular}{|c|c|c|c|c|c|}
\hline & \multicolumn{5}{|c|}{ Ratio TPM Biofilm cultures / TPM pure cultures } \\
\hline & \multicolumn{3}{|c|}{ GP59 } & \multicolumn{2}{|c|}{ NL23 } \\
\hline$\underline{\text { Genes }}$ & Ref300N-23C & $900 \mathrm{~N}-30 \mathrm{C}$ & $0 \% \mathrm{NaCl}$ & $0 \% \mathrm{NaCl}$ & Genes \\
\hline \multicolumn{6}{|l|}{ Denitrification } \\
\hline $\operatorname{narXL}$ & ns & ns & ns & -9.4 & napGH \\
\hline narK1K2GHJI-1 & ns & -7.4 & 2.1 & 8.2 & napEFDABC \\
\hline narK12 & $\mathrm{ns}$ & $\mathrm{ns}$ & 2.0 & & \\
\hline narGHJI-2 & 2.7 & 2.8 & 3.0 & & \\
\hline $\operatorname{nirK}$ & 11 & 4.7 & ns & 49 & nirKV \\
\hline norCBDQ-1 & ns & ns & -2.7 & 5.9 & nor $C B Q D E$ \\
\hline norRE & ns & ns & -3.1 & & \\
\hline nor $C B Q D-2$ & 2.4 & 3.8 & 2.8 & & \\
\hline nosRZDFYL & ns & ns & ns & 5.2 & nosRZDFYLX \\
\hline$n n r S(3)$ & ns & ns & ns & ns & $n n r S(2)$ \\
\hline$n s r R(2)$ & 2.1 & 2.5 & $\mathrm{~ns}$ & ns & $n s r R$ \\
\hline$D n r N$ & 2.3 & 2.8 & -3.0 & ns & $n n r U$ \\
\hline NOdiox (2) & ns & ns & ns & 4.0 & $n n r R$ \\
\hline
\end{tabular}

Nitrogen assimilatory pathway

\begin{tabular}{|c|c|c|c|c|c|}
\hline & & & & ns & nasTS \\
\hline $\mathrm{NO}_{3} / \mathrm{NO}_{2} \operatorname{trp}$ & 6.0 & 7.2 & 17.9 & 7.8 & $n \operatorname{tr} A B C$ \\
\hline nasAnirBD & 12 & 24 & 42 & 3.3 & nirBAnasA \\
\hline$n t r Y X$ & ns & ns & ns & ns & $n t r Y X$ \\
\hline$g \ln A$ & 2.0 & 3.1 & 5.1 & $\begin{array}{l}19 \\
-2.1\end{array}$ & $\begin{array}{l}g \ln A \\
g \ln A(3)\end{array}$ \\
\hline gltBD: GOGAT & ns & ns & $\mathrm{ns}$ & ns & gltBD \\
\hline$g \ln B$ & ns & ns & ns & 14 & $g \ln B$ \\
\hline GDH & ns & ns & ns & ns & GDH \\
\hline$g \ln D$ & ns & ns & ns & -2.9 & $g \ln D$ \\
\hline$g \ln E$ & ns & ns & ns & -3.2 & $g \ln E$ \\
\hline$g \ln K$ & 18 & 24 & 25 & 22 & $g \ln K$ \\
\hline $\mathrm{NH}_{4} \operatorname{trp}$ & 11 & 11 & 11 & 20 & $\mathrm{NH}_{4}$-trp \\
\hline$g \ln L G$ & ns & 2.1 & 5.9 & $\mathrm{~ns}$ & $g \ln L G$ \\
\hline
\end{tabular}

Data values are the ratio of the Biofilm TPM divided by the planktonic pure-culture TPM. When the ratios were $<1$, the negative inverse value $(-1 /$ ratio) was calculated. Compared to each other, the positive ratio means higher relative transcript levels in the biofilm, and negative ratio higher relative transcript level in the planktonic pure cultures. ns: no significant changes.

$g \ln A$ : glutamine synthetase; gltBD: glutamate synthase; GDH: glutamate dehydrogenase; $g \ln B$ : nitrogen regulatory protein P-II 1; $g \ln K$ : nitrogen regulatory protein P-II 2; $g \ln D$ : uridylyltransferase; $g \ln L G$ : nitrogen regulation sensor histidine kinase and response regulator; trp: transporter; Diox: dioxygenase; $n n r S$ : involved in response to NO; $n s r R$ : NO-sensitive transcriptional repressor; $\operatorname{Dnr} N$ : NO-dependent regulator; nasAnas $B D$ : assmilatory nitrate and nitrite reductase; $n t r X Y$ : Nitrogen regulation proteins; narXL: nitrate/nitrite sensor histidine kinase and response regulator; narK: nitrate/nitrite transporter; $n o s R E$ : NO- reductase transcription regulator and activation protein. 


\section{Table 6(on next page)}

Microbial diversity and the associated denitrification genes in the biofilm cultures from the de novo transcript assembly 
1 Table 6. Microbial diversity and the associated denitrification genes in the biofilm cultures from the 2 de novo transcript assembly

3

4

5

7

8

9

10

11

12

13

14

15

16

17

18

19

20

21

22

23

24

25

26

27

28

29

30

31

32

33

34

35

36

37

38

39

40

41

42

43

44

45

46

47

48

49

50

\begin{tabular}{|c|c|c|c|c|}
\hline \multirow[t]{2}{*}{ Affiliation } & \multicolumn{3}{|c|}{ Biofilm cultures (TPM) } & \multirow[b]{2}{*}{ Denitrification genes } \\
\hline & Ref300N-23C & $900 \mathrm{~N}-30 \mathrm{C}$ & $0 \% \mathrm{NaCl}$ & \\
\hline \multicolumn{5}{|l|}{ - Actinobacteria } \\
\hline Streptomyces & 6103 & 729 & 2499 & - \\
\hline \multicolumn{5}{|l|}{-Bacteroidetes } \\
\hline Sunxiuqinia & 212 & 504 & 2639 & nor $B$ \\
\hline Geofilum & 77 & 109 & 11870 & - \\
\hline Aequorivita & 18218 & 25569 & 1398 & nirK, nor $B ; C ; D ; Q$, nosZ;DFY \\
\hline Winogradskyella & 8182 & 7917 & 169 & $\operatorname{nir} K, \operatorname{nor} C, \operatorname{nos} Z ; L ; D$ \\
\hline Lentimicrobium & 3032 & 3370 & 1671 & - \\
\hline Xanthomarina & 190 & 4555 & 9598 & nirK, nor $B ; D ; C ; Q, \operatorname{nos} Z ; L ; D$ \\
\hline -Ignavibacteriales & 5513 & 14206 & 171 & nap $C ; H$ \\
\hline -Tenericutes & 6367 & 5003 & 13409 & - \\
\hline \multicolumn{5}{|l|}{ - Alphaproteobacteria } \\
\hline Aminobacter & 18 & 438 & 5385 & napA \\
\hline Aquamicrobium & 23 & 456 & 22835 & napA \\
\hline Hoeflea & 3882 & 87 & 1365 & narG;H;J;I, nosZ;R;D, nirK \\
\hline Hyphomicrobium & 0 & 1679 & 30057 & narH;I, nor $B ; C ; Q ; D ; E, \operatorname{nos} Z ; D ; R ; F$ \\
\hline Mesorhizobium & 411 & 1050 & 12505 & napA;D;E, nirK, nosZ \\
\hline Paracoccus & 1427 & 1219 & 1204 & $\operatorname{nir} S, \operatorname{nar} C ; D ; Q, \operatorname{nos} R$ \\
\hline Roseovarius & 12367 & 16479 & 686 & $\operatorname{nir} S, \operatorname{nir} K, \operatorname{nor} B ; C ; D ; Q ; E, \operatorname{nos} Z ; D ; K$ \\
\hline Stappia & 39926 & 30885 & 39745 & $\begin{array}{l}\text { nap } A B C, \text { nap } A D F E-n n r S, \text { nirK, } \\
\text { norCBQD, nosRZDF;E }\end{array}$ \\
\hline Maritimibacter & 6363 & 8360 & 10000 & narI \\
\hline Oceanibaculum & 18115 & 9071 & 25544 & $\operatorname{nar} G ; H ; J ; I$ \\
\hline \multicolumn{5}{|l|}{ - Betaproteobacteria } \\
\hline Azoarcus & 48 & 120 & 2854 & nap $A$, nos $Z$ \\
\hline \multicolumn{5}{|l|}{-Deltaproteobacteria } \\
\hline Bradymonas & 73 & 9652 & 11178 & - \\
\hline \multicolumn{5}{|l|}{ - Gammaproteobacteria } \\
\hline Marinicella & 36554 & 125466 & 14924 & $\operatorname{nir} S, \operatorname{nor} B ; C ; Q ; D$ \\
\hline Marinobacter & 14545 & 1122 & 264 & $\begin{array}{l}\text { narG;H;J;I, norB;C:Q, nirK, } \\
\text { nirS, nos } Z ; F ; D ; R\end{array}$ \\
\hline Methylophaga & 64802 & 78566 & 80482 & $\operatorname{nar} G ; H ; J ; I$, nor $B ; C ; D ; Q ; E$, nos $D ; R$ \\
\hline Idiomarina & 5147 & 1027 & 513 & narG;H;J nirK, nirS, norB \\
\hline Pseudomonas & 6175 & 1333 & 18198 & $\begin{array}{l}\operatorname{nar} G ; H ; J ; I, \operatorname{nir} K, \operatorname{nir} S, \\
\operatorname{nor} B ; C ; D ; Q, \operatorname{nos} Z ; R\end{array}$ \\
\hline Wenzhouxiangella & 132 & 2857 & 83 & - \\
\hline -Other bacteria & 113200 & 243523 & 155554 & $*$ \\
\hline •Archaea & 113 & 297 & 303 & \\
\hline •Eukarya & 181 & 352 & 416 & \\
\hline -Phages, viruses, plasmids & 99623 & 5740 & 217095 & \\
\hline -Unclassified & 281015 & 207759 & 157515 & \\
\hline - Transcripts with no genes & 248782 & 196062 & 148792 & \\
\hline
\end{tabular}

Reads that did not align to the three reference genomes were de novo assembled. These reads were then aligned to these assembled sequences. The relative transcript levels of the assembled sequences in a metatranscriptome were expressed as transcripts per million (TPM). Putative genes from the assembled 
51 sequences were annotated for function and affiliation. The TPM of the genes affiliated to specific bacteria 52 taxa were then summed. Denitrification genes identified by annotations from respective affiliated bacterial 53 taxa were sorted out. * Denitrification genes were found scattered in other bacterial taxa. 